\title{
RESEARCH
}

Open Access

\section{Diet-induced remission in chronic enteropathy is associated with altered microbial community structure and synthesis of secondary bile acids}

Shuai Wang ${ }^{1}$, Rene Martins², Megan C. Sullivan', Elliot S. Friedman³ , Ana M. Misic' ${ }^{1}$, Ayah El-Fahmawi', Elaine Cristina Pereira De Martinis ${ }^{4}$, Kevin O'Brien ${ }^{1}$, Ying Chen ${ }^{1}$, Charles Bradley ${ }^{1}$, Grace Zhang ${ }^{1}$,

Alexander S. F. Berry ${ }^{1,5}$, Christopher A. Hunter ${ }^{1}$, Robert N. Baldassano ${ }^{5}$, Mark P. Rondeau ${ }^{2}$ and Daniel P. Beiting ${ }^{1^{*}}$ (D)

\begin{abstract}
Background: The microbiome has been implicated in the initiation and persistence of inflammatory bowel disease. Despite the fact that diet is one of the most potent modulators of microbiome composition and function and that dietary intervention is the first-line therapy for treating pediatric Crohn's disease, the relationships between dietinduced remission, enteropathy, and microbiome are poorly understood. Here, we leverage a naturally-occurring canine model of chronic inflammatory enteropathy that exhibits robust remission following nutritional therapy, to perform a longitudinal study that integrates clinical monitoring, 165 rRNA gene amplicon sequencing, metagenomic sequencing, metabolomic profiling, and whole genome sequencing to investigate the relationship between therapeutic diet, microbiome, and disease.

Results: We show that remission induced by a hydrolyzed protein diet is accompanied by alterations in microbial community structure marked by decreased abundance of pathobionts (e.g., Escherichia coli and Clostridium perfringens), reduced severity of dysbiosis, and increased levels of the secondary bile acids, lithocholic and deoxycholic acid. Physiologic levels of these bile acids inhibited the growth of E. coli and C. perfringens isolates, in vitro. Metagenomic analysis and whole genome sequencing identified the bile acid producer Clostridium hiranonis as elevated after dietary therapy and a likely source of secondary bile acids during remission. When C. hiranonis was administered to mice, levels of deoxycholic acid were preserved and pathology associated with DSS colitis was ameliorated. Finally, a closely related bile acid producer, Clostridium scindens, was associated with diet-induced remission in human pediatric Crohn's disease.

Conclusions: These data highlight that remission induced by a hydrolyzed protein diet is associated with improved microbiota structure, an expansion of bile acid-producing clostridia, and increased levels of secondary bile acids. Our observations from clinical studies of exclusive enteral nutrition in human Crohn's disease, along with our in vitro inhibition assays and in vivo studies in mice, suggest that this may be a conserved response to diet therapy with the potential to ameliorate disease. These findings provide insight into diet-induced remission of gastrointestinal disease and could help guide the rational design of more effective therapeutic diets.
\end{abstract}

Keywords: Chronic enteropathy, Dietary therapy, Microbiome, Bile acids, Canine, Metabolomics, Crohn's disease

\footnotetext{
* Correspondence: beiting@upenn.edu

'Department of Pathobiology, School of Veterinary Medicine, University of

Pennsylvania, Philadelphia, PA 19104, USA

Full list of author information is available at the end of the article
}

(c) The Author(s). 2019 Open Access This article is distributed under the terms of the Creative Commons Attribution 4.0 International License (http://creativecommons.org/licenses/by/4.0/), which permits unrestricted use, distribution, and reproduction in any medium, provided you give appropriate credit to the original author(s) and the source, provide a link to the Creative Commons license, and indicate if changes were made. The Creative Commons Public Domain Dedication waiver (http://creativecommons.org/publicdomain/zero/1.0/) applies to the data made available in this article, unless otherwise stated. 


\section{Background}

Human inflammatory bowel disease (IBD), including Crohn's disease and ulcerative colitis, is a multifactorial and debilitating disease characterized by chronic immunepathology, disruption of intestinal homeostasis, and altered composition of the gut microbiome (dysbiosis). Several lines of evidence point to resident gut bacteria as important factors in the etiology of IBD. First, disease is often more severe in areas of the intestine with the highest microbial biomass, and antibiotics are frequently used as an adjunct therapy with immunosuppressants or monoclonal antibodies for managing IBD [1, 2]. Second, genome-wide association studies have identified numerous susceptibility loci in genes responsible for recognizing or responding to bacteria [3]. Finally, in some mouse models of colitis, disease can be transferred to naive hosts via fecal transplant [4-6], suggesting a causal role for gut microbes in disease. Collectively, these findings have led to a "two-hit" model for IBD in which both host genetics and microbial factors influence disease presentation, highlighting an opportunity to develop novel microbiome-based treatments for IBD.

Although a range of environmental factors have been shown to influence the microbiome, diet is regarded as one of the most potent modulators of the composition and function of the gut-resident microbial community in healthy humans and other mammals [7, 8], and can act as both a risk factor and a treatment modality for IBD $[9,10]$. Epidemiologic data and studies in mice have shown that diets high in fat and/or low in fiber, as well as dietary additives such as emulsifiers, are either risk factors for IBD, or in some cases can directly compromise intestinal barrier function leading to disease [11-13]. Diet can be also leveraged to treat IBD, with perhaps the clearest example of this being the use of exclusive enteral nutrition (EEN) as first-line therapy for pediatric Crohn's disease [14]. High remission rates $(\geq 60 \%)$ are observed following EEN, and compared to corticosteroids, EEN achieves better patient growth along with a reduction in biomarkers of disease, such as fecal calprotectin and C-reactive protein [15-18]. Interestingly, EEN has a marked effect on the microbiota community, but the precise nature of this effect has been complicated to discern, with some studies reporting reduced microbiome diversity following EEN therapy [19-21], while others point to relatively unchanged [22, 23] or increased diversity [24].

The relationship between diet, microbiome structure, and enteropathy is complicated to dissect from human subject research where diet is challenging to control, necessitating either retrospective studies in conjunction with extensive food intake surveys [25], controlled feeding studies [26], or focusing on populations with different subsistence practices [27-29]. While mouse models have yielded important insights into the pathophysiology of colitis, they often involve chemical or genetic perturbation, rather than spontaneous disease development. Moreover, the ubiquitous use of autoclaved food and acidified water for mouse husbandry, together with the tendency for cage effects to dominate in mouse microbiome studies, raises concerns about clinical relevance of diet-microbiome studies in murine models of colitis. A growing number of studies suggest that dogs are an ideal animal model in which to study translationally relevant diet-microbiome interactions in the context of intestinal disease. First, as companion animals, dogs share the same environment with humans and spontaneously develop a chronic enteropathy (CE) that clinically resembles some aspects of human IBD, including several shared features of gastrointestinal pathology, responsiveness to similar treatments [30,31], involving some of the same susceptibility loci [30-32], and shared disease-associated microbial taxa [33-35]. Secondly, a recent metagenomic study produced a catalog of over one million taxonomically and functionally annotated microbial genes from the canine gut and showed that-compared to other mammals, such as the mouse and pig-the microbial environment in dogs most closely resembles that of humans [36]. Thirdly, the canine microbiome was markedly altered by diets differing in protein/carbohydrates in a manner that resembles what has been reported in humans [36]. As treatment with dietary therapy promotes a long-lasting state of remission in over 50\% of dogs with CE [37], this animal model provides an opportunity to rigorously investigate the relationship between therapeutic diet, microbiome, and disease resolution.

Despite the fact that the gut microbiome has been implicated in IBD pathogenesis and that diet can be used to manage symptoms of IBD, there is limited insight into how this occurs. In this study, we examined dogs with $\mathrm{CE}$ and monitored changes in their fecal microbial community structure and metabolites in response to treatment with a therapeutic hydrolyzed protein diet. By comparing changes over time in dogs with food-responsive chronic enteropathy (FRE), versus animals that failed diet therapy and required subsequent combination therapy, we showed that the hydrolyzed protein diet induces rapid remission that is associated with a structural and functional remodeling of the microbial community in the gut. Notably, we observed that secondary bile acids, likely produced by $C$. hiranonis, are associated with the diet-induced remission of canine $C E$ and exert antibacterial activity against isolates of $E$. coli and C. perfringens in vitro, suggesting these metabolites may modulate disease. These findings improve our understanding of how dietary therapy can modulate microbial communities and reduce GI disease.

\section{Results}

Dietary therapy induces rapid and durable remission Twenty-nine dogs with $\mathrm{CE}$ were enrolled in a study to evaluate the Effect of Nutritional Therapy on Microbiome 
in Canine Enteropathy (the "ENTiCE" study). Animals with active disease were switched from their current diet to a commercially available therapeutic hydrolyzed protein diet (Fig. 1a). Impact of treatment on disease was monitored using the Canine Chronic Enteropathy Clinical Activity Index (CCECAI; hereafter referred to as "disease score"), which is positively correlated with poor clinical outcome [37]. After 2 weeks on therapeutic diet, 69\% (20/ 29) of animals entered remission, marked by a reduction in the mean disease score from $4.1(95 \% \mathrm{CI}=4.8-3.3)$ to $1.3(95 \% \mathrm{CI}=1.8-0.7)$. These diet-responsive (DR) animals (dogs with FRE) were maintained on diet for the remainder of the study with no additional interventions (Fig. 1a). At the conclusion of the study (day 42), DR animals had a mean disease score of $0.9(95 \% \mathrm{CI}=1.3$ $0.6)$, constituting a $>4$-fold reduction in disease severity compared to day 0 (Fig. 1b). In contrast, 31\% (9/29) of animals failed to show a significant reduction in disease score after 2 weeks on therapeutic diet (Fig. 1c). These non-diet-responsive (NDR) animals presented with more severe disease scores (mean score $=6.1 ; 95 \% \mathrm{CI}=$ 7.4-4.7) than DR animals $(P<0.05$ at day 0$)$ and did not show a significant reduction 2 weeks after starting diet therapy (Fig. 1c). NDR animals were maintained on therapeutic diet for the remainder of the study, while receiving combination therapy that included antibiotics (beginning at day 14) and prednisone (beginning at day 28) (Fig. 1a and Additional file 1: Figure S1, see the "Methods" section), but showed only incremental improvement in disease scores (Fig. 1c). These data highlight a rapid and sustained clinical response to hydrolyzed diet in the majority of dogs with CE.

\section{Identification of microbial community profiles associated with treatment outcome}

To determine whether treatment with hydrolyzed diet alone altered the microbial community in the gut, $16 \mathrm{~S}$ rRNA gene profiling was carried out on fecal samples collected from DR $(n=20)$, NDR $(n=9)$, and healthy control animals at baseline $(n=24)$. Consistent with previous reports [38], we found that the species diversity of the canine fecal microbiome was not dramatically altered in dogs with CE compared to healthy controls (Additional file 1: Figure S2A-B) and that the communities in both groups were predominantly comprised of Firmicutes, Bacteroidetes, Proteobacteria, Actinobacteria, and Fusobacteria (Additional file 1: Figure S2C). However, compared to healthy dogs, animals with $\mathrm{CE}$ showed greater between-individual distance in microbial community structure by weighted UniFrac (Additional file 1 : Figure S2D). Using a ternary plot visualization, we observed an enrichment of operational taxonomic units (OTUs) from Proteobacteria and a subset of OTUs from Firmicutes in animals with active disease at day 0
(Fig. 1d, green and tan points toward left side of triangle; Additional file 1: Figure S3). Interestingly, a subset of proteobacterial OTUs was highly enriched in DR animals compared to both NDR and healthy controls (Fig. 1d, tan points in lower left corner).

These differences prompted us to carry out a formal differential abundance analysis, identifying 84 OTUs that distinguish healthy animals from those with disease (Additional file 2: Table S1 and Additional file 1: Figure S3). For example, Escherichia coli, which is commonly associated with intestinal disease, was overrepresented in animals with CE (Fig. 1e), showing a significant, albeit weak, positive correlation with disease score $(R=0.22$, $P=0.02$ ) (Fig. 1f). OTUs from Clostridium sensu stricto 1 were also enriched in $\mathrm{CE}$, including Clostridium perfringens (Fig. 1g), which was also positively correlated with disease scores (Fig. 1h) $(R=0.23, P=0.016)$ and which has been implicated in large bowel diarrhea/colitis in dogs [39]. Taken together with previously published work [40,41], these data demonstrate that dysbiosis during $C E$ is marked by the presence of pathobionts, such as $E$. coli and C. perfringens. Next, we asked whether the microbiome in DR and NDR animals differed prior to starting treatment (day 0). Although no differences were observed between the two groups in community diversity, evenness, or distance from healthy controls (unweighted or weighted UniFrac), we identified 20 OTUs that were differentially abundant between DR and NDR animals, 12 of which were enriched in animals that ended up responding to diet treatment (Fig. 1i and Additional file 2: Table S2). Interestingly, Proteobacteria were found to be more abundant in DR animals (Fig. 1i). Collectively, these results reveal distinct microbial signatures during disease that are associated with different clinical outcomes following the dietary therapy.

\section{Therapeutic diet reduces dysbiosis associated with chronic enteropathy}

To assess whether the diet-induced remission was accompanied by alterations in dysbiosis, we compared the microbial community structure before and after administration of therapeutic diet in DR animals. Although global phylogenetic distance and Shannon diversity were unchanged (Additional file 1: Figure S4A-B), a measurable increase in community evenness was observed following 14 days of diet therapy (Fig. 2a and Additional file 1: Figure S5), when focusing on the top 40 most abundant OTUs among the samples, which account for $83 \%$ of the total reads. Principal coordinate analysis ( $\mathrm{PCOA})$ based on unweighted (Additional file 1: Figure S4C) or weighted (Additional file 1: Figure S4D) UniFrac showed a measurable separation between dogs, even at day 0, before diet was administered. Despite this baseline difference between animals, community structure underwent a shift away from disease state by 


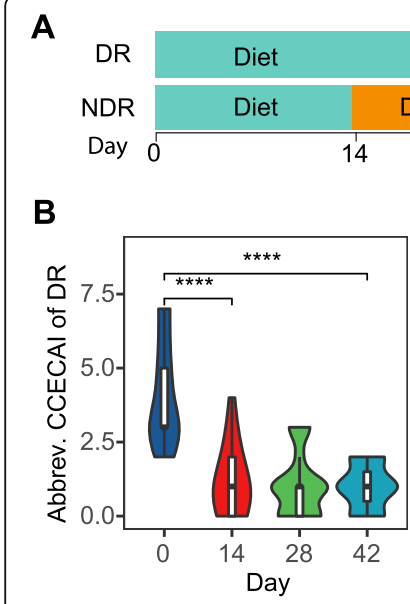

\begin{tabular}{|c|c|c|c|}
\hline DR & Diet & Diet & Diet \\
\hline NDR & Diet & Diet + Abtx & Diet + Abtx+ Pred \\
\hline Day 0 & & & 42 \\
\hline
\end{tabular}

D
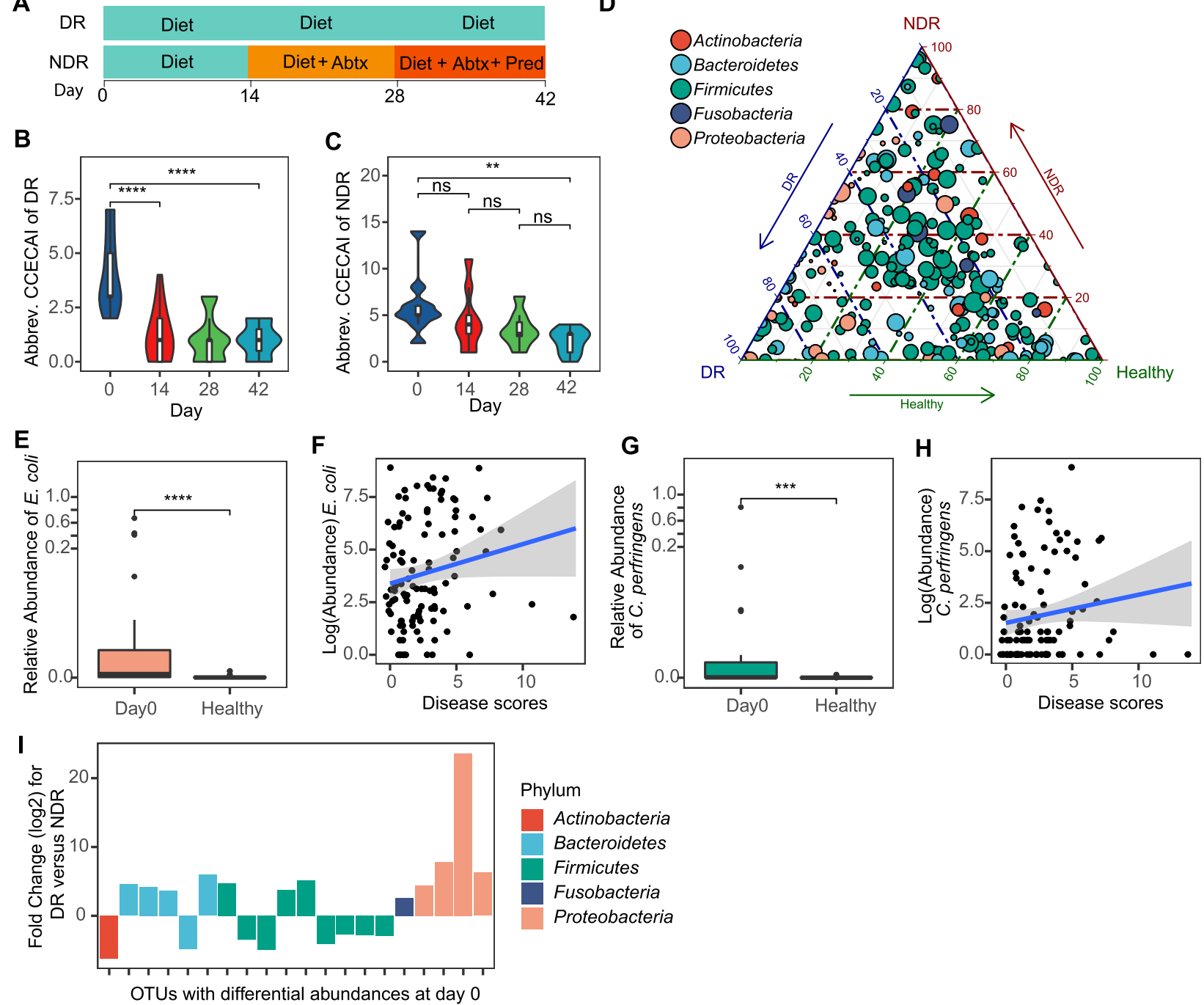

Fig. 1 Identification of microbial community profiles associated with treatment outcome in diet therapy. a Schematic showing clinical study design for identifying diet-responsive (DR) and non-diet-responsive (NDR) dogs. Antibiotics (Abtx) and prednisone (Pred) treatments are indicated. Abbreviated Canine Chronic Enteropathy Clinical Activity Index (CCECAl) scores were assessed at four different time points in $\mathbf{b}$ DR $(n=20)$ and $\mathbf{c}$ NDR $(n=9)$ animals. $\mathbf{d}$ Ternary plot of phylum-level OTUs from top 5 most abundant phyla among healthy (right), DR (left), and NDR (top) animals. Only OTUs with a maximum relative abundance $>0.001$ and existing in at least $10 \%$ samples are shown. Bubble size represents the log2transformed mean abundance of each OTU. Axes show reads accounted for by each OTU in each group of animals (DR, NDR, and healthy), as a percentage of total (sum) reads observed for a given OTU across all three groups. Arrows indicate the corresponding axis directions for each point. Relative abundance of $\mathbf{e} E$. coli and $\mathbf{g}$ C. perfringens in animals with active disease (day 0 ) and healthy dogs. Spearman's correlation between $\log 10$ abundance of $\mathbf{f} E$. coli or $\mathbf{h}$ C. perfringens and CCECAl disease score. The shaded areas show the $95 \%$ confidence interval, as implemented in the geom_smooth function in the ggplot2 R package. i Differentially abundant OTUs between DR and NDR animals at day $0 . Y$ axis value represents the log2 fold change for DR versus NDR. ${ }^{*} P<0.05,{ }^{* *} P<0.01,{ }^{* * *} P<0.001,{ }^{* * *} P<0.0001$ using the two-sided Wilcoxon signed-rank test. Spearman's correlations in $\mathbf{f}$ and $\mathbf{h}$ are significant $(P<0.05)$ with correlation coefficients of 0.216 and 0.227 , respectively

14 and 42 days after diet therapy (Additional file 1: Figure S4C and D). This shift is significantly higher than the heterogeneity within healthy group, suggesting an influence from diet therapy (Additional file 1: Figure S4E). Comparing weighted (Fig. 2b) or unweighted (Additional file 1: Figure S4F) UniFrac distances between DR and healthy animals at each time point, we observed that diet-induced remission was accompanied by decreased distance relative to healthy controls, a trend that continued through day 42, when the similarity to day 0 was lowest and similarity to healthy dogs was highest (Fig. 2b).

Given that therapeutic diet is associated with a shift in community structure of the microbiome in DR animals, we reasoned that composition of the fecal microbiome would be rapidly altered after dietary intervention. Administration of therapeutic diet was broadly characterized by 


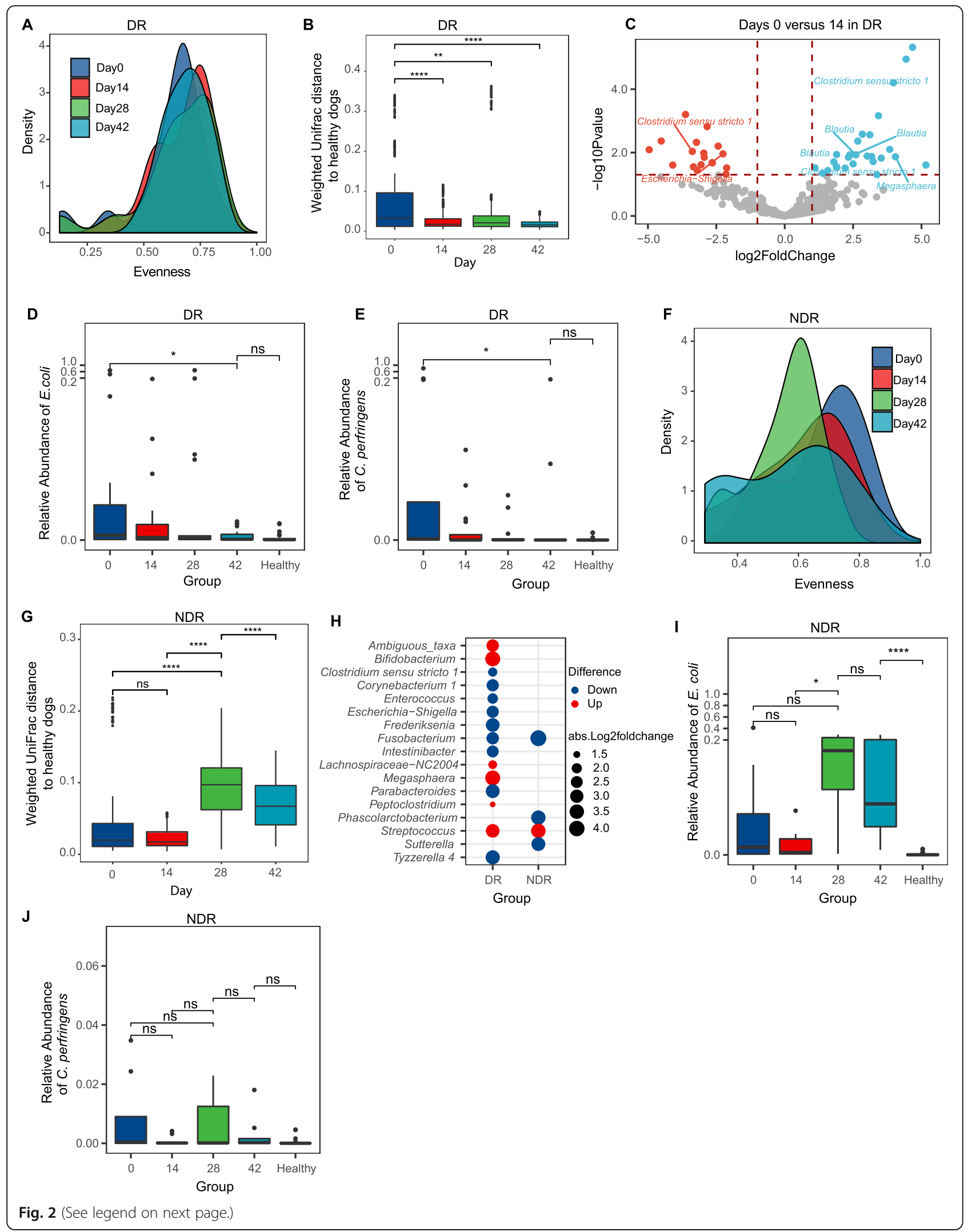




\section{(See figure on previous page.)}

Fig. 2 Therapeutic diet ameliorates dysbiosis associated with chronic enteropathy and induces changes in the microbiome associated with remission. a Density of Pielou's evenness index for DR animals at different time points in the study. $\mathbf{b}$ Weighted UniFrac distance to healthy controls for DR animals. c Volcano plot showing differentially abundant OTUs enriched in either DR dogs with active disease (day 0, red points) or in remission after diet therapy (day 14, blue points). Selected taxa (e.g., Escherichia-Shigella spp., Clostridium spp., and Blautia; OTUs with mean abundance $>5$ counts) are labeled. The relative abundance of $\mathbf{d} E$. coli and $\mathbf{e} C$. perfringens in DR animals throughout the study and compared to healthy controls. $\mathbf{f}$ Density of Pielou's evenness index in NDR animals. $\mathbf{g}$ Weighted UniFrac distance to healthy dogs for NDR animals. Diet therapy began at day 0 , metronidazole administration at day 14, and prednisone at day 28 (see the "Methods"). $\mathbf{h}$ Bubble plot showing differentially abundant genera (fold change $>2$ and $P<0.05$ ) between day 14 versus day 0 for DR (left) and NDR (right) animals. Bubble size indicates absolute log fold change between day 14 and day 0 , and color reflects direction of change. $\mathbf{i}$, $\mathbf{j}$ The relative abundance of $E$. coli and C. perfringens in NDR animals throughout the study and compared to healthy controls. The distribution comparison of density ( $\mathbf{a}, \mathbf{f}$ ) was performed by comparing quantiles via the Harrell-Davis estimator (FDR $<0.01$ ). ns, not significant. ${ }^{*} P<0.05$, ${ }^{* *} P<0.01$, ${ }^{* * *} P<0.001$, ${ }^{* * *} P<0.0001$ using the two-sided Wilcoxon rank sum test for $\mathbf{b}$ and $\mathbf{g}$ or using the two-sided Wilcoxon signed-rank sum test for panels $\mathbf{d}, \mathbf{e}, \mathbf{i}$, and $\mathbf{j}$

an increase of Firmicutes and a decrease of Proteobacteria (Additional file 1: Figure S4G and H). Fourteen days after beginning diet therapy, 14 genera were differentially abundant compared to pretreatment (day 0) in DR animals (Additional file 2: Table S3). Potentially pathogenic genera associated with IBD were underrepresented after diet treatment in DR animals. For example, Escherichia-Shigella, Clostridium sensu stricto 1, Enterococcus, and Fusobacterium had a higher relative abundance at day 0 and were significantly reduced after 14 days on therapeutic diet. When evaluated at the species level, 45 OTUs were significantly differentially abundant between samples collected at day 0 compared to day 14 (Fig. 2c, Additional file 2: Table S4). E. coli was enriched in animals at day 0 in this study (Fig. 2d), and relative abundance of this taxon declined dramatically after 2 weeks on therapeutic diet, eventually reaching levels nearly undetectable by day 42 that were also indistinguishable from levels observed in healthy dogs (Fig. 2d). C. perfringens also showed a significantly lower prevalence in the samples at day 14 and in healthy dogs, compared to day 0 samples (Fig. 2e). In contrast, OTUs from Blautia were increased after diet therapy, consistent with reports that members of this genus are beneficial commensals [42] (Additional file 2: Table S4). Taken together, these results point to ameliorated dysbiosis with a reduction of pathobionts and increase of beneficial commensal taxa as a hallmark of the diet therapy.

\section{Remission-specific changes in the microbiome following diet therapy}

We hypothesized that the changes observed following diet therapy in DR animals would be associated with remission, rather than merely a response to diet that is independent of clinical outcome. To test this hypothesis, we compared the impact of therapeutic diet on DR dogs with changes observed between days 0 and 14 in NDR animals that failed to enter remission after diet therapy, and which required additional therapies after day 14 . Whereas 14 days of diet therapy in DR animals was associated with increased community evenness (Fig. 2a) and a decreased distance from healthy dogs (Fig. 2b), the same treatment in NDR dogs did not significantly affect the microbial community evenness (Fig. 2f, red) or weighted (or unweighted) UniFrac distance to healthy dogs (Fig. 2g and Additional file 1: Figure S4I). Just as we observed in DR animals (Additional file 1: Figure S4G), diet also altered the gut microbiota compositions in NDR animals (Additional file 1: Figure S4J). However, this shift was distinct from that observed in DR animals (Fig. 2h). Differential abundance analysis comparing NDR animals at day 0 versus day 14, during which time they received only therapeutic diet, identified 23 OTUs (Additional file 2: Table S5). Diet therapy in NDR animals was associated with a decrease in the relative abundance of some taxa, such as Fusobacterium and Phascolarctobacterium taxa, that were either unchanged or more modestly altered by diet therapy in DR animals. Conversely, Escherichia-Shigella, Enterococcus, and some of Clostridium sensu stricto 1 were not reduced in animals that failed diet therapy (Fig. 2h). Similarly, E. coli and $C$. perfringens were not significantly reduced in NDR animals after diet therapy (Fig. 2i, j). After 14 days on therapeutic diet, NDR dogs were maintained on diet, but were also administered metronidazole, an antibiotic that largely targets anaerobes. As expected, antibiotic treatment exacerbated dysbiosis, resulting in a precipitous decline in community evenness (Fig. 2f, green), increased distance from healthy controls (Fig. 2g, green), and increased relative abundance of $E$. coli and C. perfringens (Fig. 2i, j, green). Interestingly, steroid treatment (initiated on day 28 for NDR animals) mildly ameliorated the dysbiosis, resulting in decreased distance to healthy dogs (Fig. 2g, light blue).

\section{Diet-induced remission is associated with metabolic reprogramming and increased levels of secondary bile acids}

To determine if the changes in microbial community structure would translate to altered microbial metabolism, stool samples collected day 0,14 , and 42 after starting diet therapy were subjected to metagenomic sequencing. Principal 
component analysis of metabolic pathway abundance data showed a separation $(P<0.05)$ between DR animals before and after diet-induced remission (days 0 vs. 14) (Fig. 3a, b). Differential abundance analysis identified 35 global pathways and 120 pathways at the species level that were significantly altered in relative abundance as a result of diet treatment in DR animals (Additional file 2: Table S6), including several involved in carbohydrate metabolism and secondary bile acid synthesis, prompting us to quantify bile acids in stool. Using targeted metabolomics, we measured the levels of 15 bile acids in stool of healthy dogs, compared with days 0, 14, and 42 (Additional file 1: Figure S6). Consistent with increased relative abundance of bile salt hydrolase (BSH) and bile acid-inducible operon (bai) genes (Fig. 3c and Additional file 1: Figure S7) involved in secondary bile acid synthesis (Fig. 3d), the secondary bile acids deoxycholic acid (Fig. 3e) and lithocholic acid (Fig. 3f) were reduced in animals with active disease (day 0), compared to healthy dogs, and were significantly increased after the diet treatment in DR animals at day 14 and 42 (Fig. 3e, f, and Additional file 2: Table S7). Notably, secondary bile acids were not elevated by diet treatment in NDR animals (Fig. 3g, h), suggesting that this metabolic shift is associated with remission.

\section{Lithocholic and deoxycholic acid inhibit the in vitro growth of $E$. coli and C. perfringens}

Given that bile acids have emerged as potential regulators of the gut microbiome, we hypothesized that the secondary bile acids observed in our study could impact microbiome structure [43]. Correlation analysis of our metabolomics and microbiome data reveal that the primary bile acid, cholic acid, was negatively correlated with 10 OTUs (Additional file 2: Table S8), consistent with the reported ability of this bile acid to broadly and negatively regulate bacterial growth [43]. We also observed that the increase in secondary bile acids following diet treatment correlated with reduced relative abundance of certain bacteria (e.g., OTUs from Escherichia-Shigella, Clostridium, and Fusobacterium) (Additional file 2: Table S8). To directly test whether secondary bile acids could have a negative impact on potential pathogenic bacteria from the canine gut, lithocholic and deoxycholic acid were assessed for their ability to inhibit the in vitro growth of E. coli $(n=1)$ or $C$. perfringens $(n=3)$ isolates derived from dogs with active CE in our ENTiCE study. These species were selected since either they or their parent genera were associated with disease in our animal model. Deoxycholic acid blocked the growth of both species at a concentration comparable to what we detected in the fecal samples (in DR animals at day 42, mean $=0.43 ; 95 \%$ CI 0 . $22-0.63 \mathrm{mg} / \mathrm{g}$ ) (Fig. 4b and Additional file 1: Figure S6), while lithocholic acid (in DR animals at day 42, mean = 0.09; $95 \%$ CI $0.05-0.13 \mathrm{mg} / \mathrm{g}$ ) modestly blocked the growth of $E$. coli but not C. perfringens (Fig. 4a). In contrast, C. perfringens is much more sensitive to deoxycholic acid than $E$. coli (Fig. 4c, d). Collectively, these results show that the inhibitory activity of these secondary bile acids varies for different bacteria and suggest that elevated lithocholic and deoxycholic acids observed following the dietary therapy may contribute to the control of harmful bacteria.

\section{C. hiranonis is a diet-responsive species with the ability to produce secondary bile acids}

Next, we sought to identify potential sources of lithocholic and deoxycholic acids after diet treatment. Conversion of primary bile acids to these secondary bile acids requires the 7-dehydroxylation activity conferred by the bile acidinducible (bai) operon-an activity unique to a limited number of anaerobes representing a small fraction of the microbiome, including some Clostridial and Eubacterial species [44]. Given our finding that certain clostridial (or pepto-clostridial) OTUs, as well as levels of lithocholic and deoxycholic acids, increase after diet-induced remission, we set out to identify potential bile acid producers in DR animals. Taxonomic assignment of metagenomic reads identified six Clostridium species (C. perfringens, $C$. hiranonis, C. nexile, C. colicanis, C. glycolicum, and C. ramosum) and two Eubacterium species (Eubacterium biforme and E. dolichum) present in these samples at a relative abundance $\geq 0.01 \%$ in at least $10 \%$ samples. Of these species, $C$. hiranonis was among the most abundant (Additional file 1: Figure S8) and is the only one reported to have the bai operon [45]. Moreover, our metagenomic data (Additional file 1: Figure S9) showed that the relative abundance of $C$. hiranonis was significantly increased after diet treatment in DR animals (Fig. 5a, left) but not in NDR animals that failed diet therapy (Fig. $5 \mathrm{a}$, right). Since the Clostridium genus exhibits a high level of genetic divergence, even at the species level, we set out to confirm that canine $C$. hiranonis possesses the bai operon. Anaerobic culture of rectal swabs collected during the study, followed by random isolate picking and Sanger sequencing of full-length $16 \mathrm{~S}$ rRNA gene, was used to assemble a canine culture collection from 7 dogs with CE before and/ or after treatment. In total, 49 Clostridium isolates belonging to 5 species were identified (C. baratii, C. perfringens, C. sartagoforme, C. hiranonis, and C. lactatifermentans). Eighty-two percent (31/39) of the clostridial isolates from animals with active disease were $C$. perfringens, consistent with the reported involvement of this organism in canine [39] and human [46] gastrointestinal disease. Two C. hiranonis isolates were obtained from independent DR animals in remission at day 42 . We selected these $C$. hiranonis isolates and three C. perfringens isolates for whole genome sequencing. Reads were aligned to the reference $C$. hiranonis genome, revealing an intact bai operon in canine $C$. hiranonis, but not $C$. perfringens 

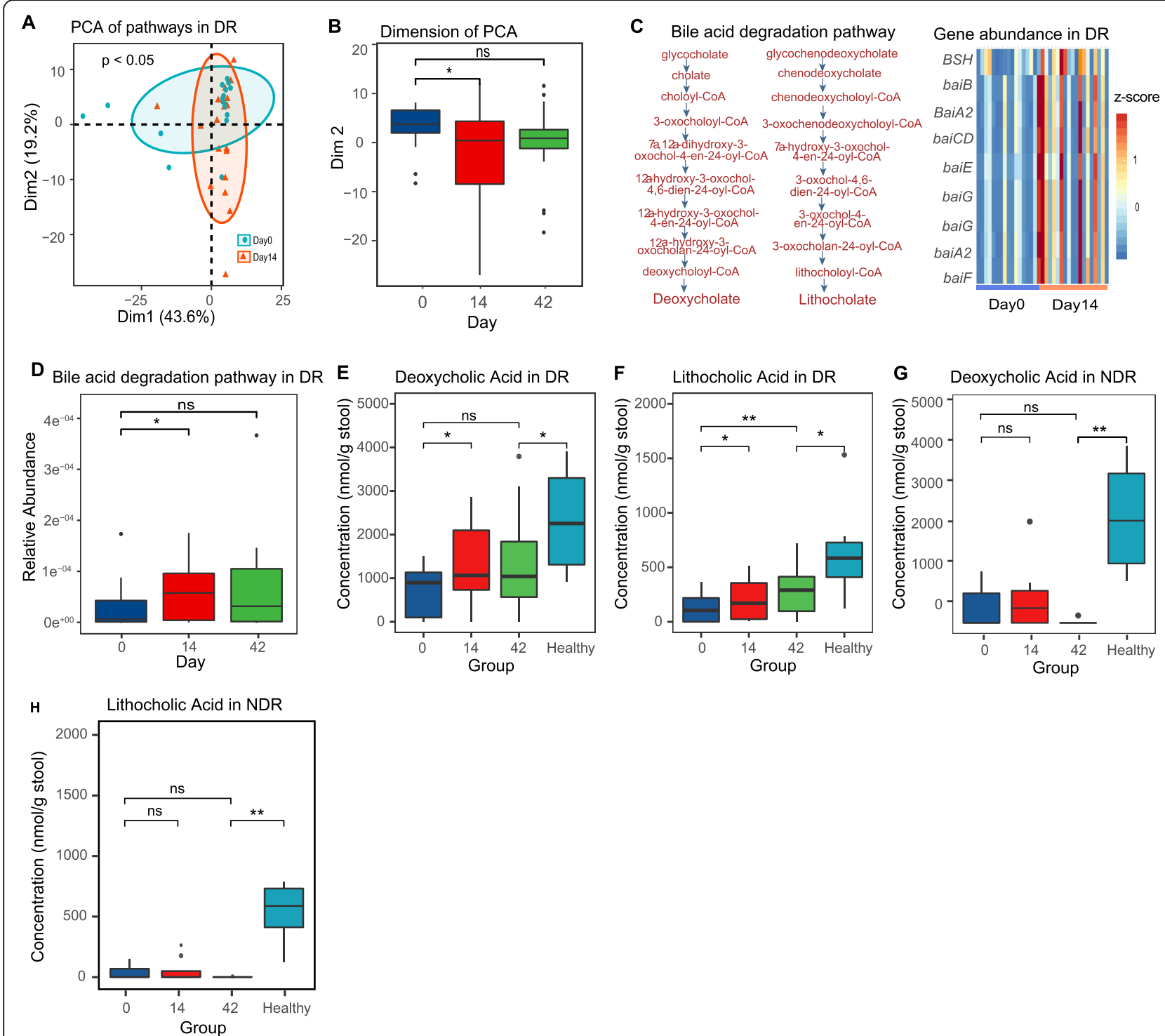

Fig. 3 Diet-induced remission is associated with metabolic reprogramming and increased levels of secondary bile acids. a PCA analysis of UniPathway pathways based on the results of HUMAnN2 analysis for DR animals. $\mathbf{b}$ The first principal component (dim 2) from $\mathbf{a}$, for all time points. c Heatmap showing the relative abundances of genes in secondary bile acid biosynthesis pathway after diet in DR animals. $\mathbf{d}$ Relative abundance of the pathway for secondary bile acid biosynthesis, predicted based on metagenomic data for DR animals. e-h Levels of deoxycholic (e) and lithocholic (f) acid measured in the stool of DR animals and NDR animals $(\mathbf{g}, \mathbf{h})$. ns, not significant. ${ }^{*} P<0.05$, ${ }^{* *} P<0.01$ using Hotelling's Tsquared test for a or the two-sided Wilcoxon signed-rank test for other panels

(Fig. 5b). In addition, the reduced abundance of C. hiranonis (Fig. 5a) after antibiotic treatment in NDR group was associated with a decrease in the level of secondary bile acids (Fig. 3g, h) in stool of these animals. As an important component in bile acid degradation, bile salt hydrolases (BSHs) [47] were examined. Thirty-one BSH-containing species were identified in our metagenomic data, the majority of which belong to the genera Lactobacillus, Streptococcus, and Eubacterium (Fig. 5c). Several of these species are significantly positively correlated with the lithocholic acid or deoxycholic acid (Additional file 2: Table S9 and Additional file 1: Figure S7B), highlighting that BSH-containing microbes may contribute to increased levels of these bile acids. Collectively, these data point to $C$. hiranonis, a species originally isolated from human stool [45], as the most likely secondary bile acid producer associated with the diet-induced remission in dogs.

The association between $C$. hiranonis, secondary bile acids, and remission in CE prompted us to test whether C. hiranonis could ameliorate disease in a mouse model 


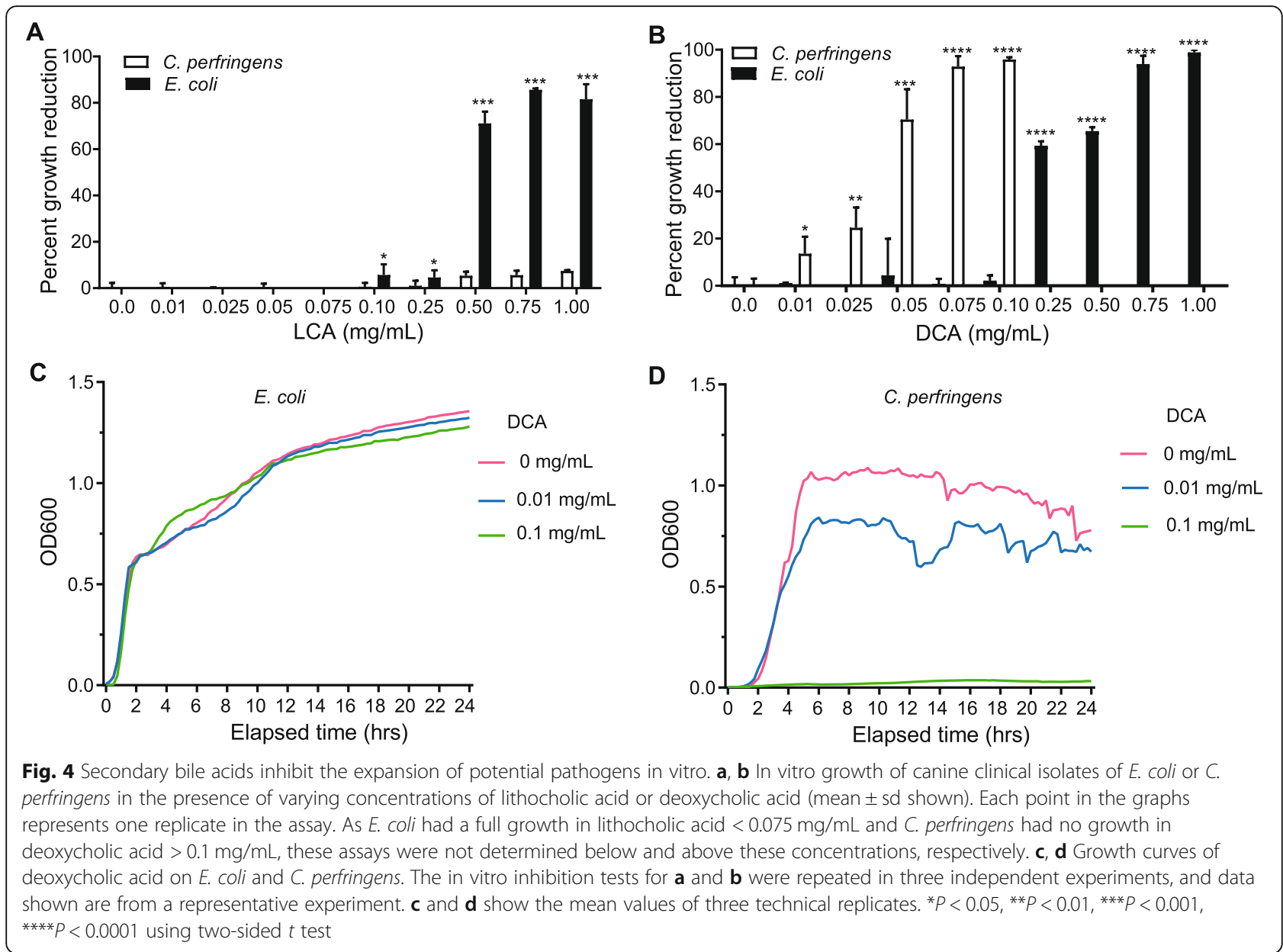

of colitis. Inflammation and dysbiosis were triggered by the administration of dextran sodium sulfate (DSS) in drinking water [48], and animals were either orally administered PBS or C. hiranonis (Fig. 5d). As expected, DSS treatment resulted in significantly reduced weight (Fig. 5e), colon length (Fig. 5f), gut damage marked by infiltration of mononuclear cells and neutrophils into the lamina propria (Fig. 5g, h; Additional file 1: Figure S10), and hyperplastic crypts with increased numbers of mitotic figures and reduced goblet cell numbers (Fig. 5g, i). In addition, DSS treatment resulted in bile acid dysregulation marked by reduced levels of deoxycholic acid in the stool (Fig. 5j). In contrast, DSS-treated mice that received $C$. hiranonis showed a marked reduction of colonic shortening (Fig. 5f), a less severe inflammation (Fig. 5g, h) that was restricted to the lamina propria and which involved fewer neutrophils, a maintenance of goblet cell numbers (Fig. 5g, i), and a preservation of deoxycholic acid levels (Fig. 5j). In addition, when mice were precolonized with a food-safe strain of $E$. coli (Nissle 1917) at day -3, DSS treatment resulted in significant bacterial expansion (dysbiosis) in mice by day 8 (Fig. $5 \mathrm{k}$ ). This expansion was markedly reduced in mice that received $C$. hiranonis. Taken together with the results from the canine model, these data suggest that $C$. hiranonis is a beneficial microbe implicated in remission of intestinal disease.

\section{C. scindens is associated with diet-induced remission in pediatric Crohn's disease}

Given that high remission rates are observed in both dogs and humans following diet therapy, we hypothesized that a similar induction of bai operon-containing clostridia may occur in pediatric Crohn's disease patients being treated with exclusive enteral nutrition (EEN). To test this, we analyzed publicly available data from the Pediatric Longitudinal Study of Elemental Diet and Stool Microbiome Composition ("PLEASE"), a study that examined approximately 20 patients before and after treatment with EEN [23], in which half responded to treatment while the other half failed EEN therapy. Classifications of bacterial taxa present in each sample using metagenomic analysis methods [49] revealed the presence of $C$. scindens, which like $C$. hiranonis is also recognized for having high 7-dehydroxylation activity $[45,50]$. The relative abundance of 


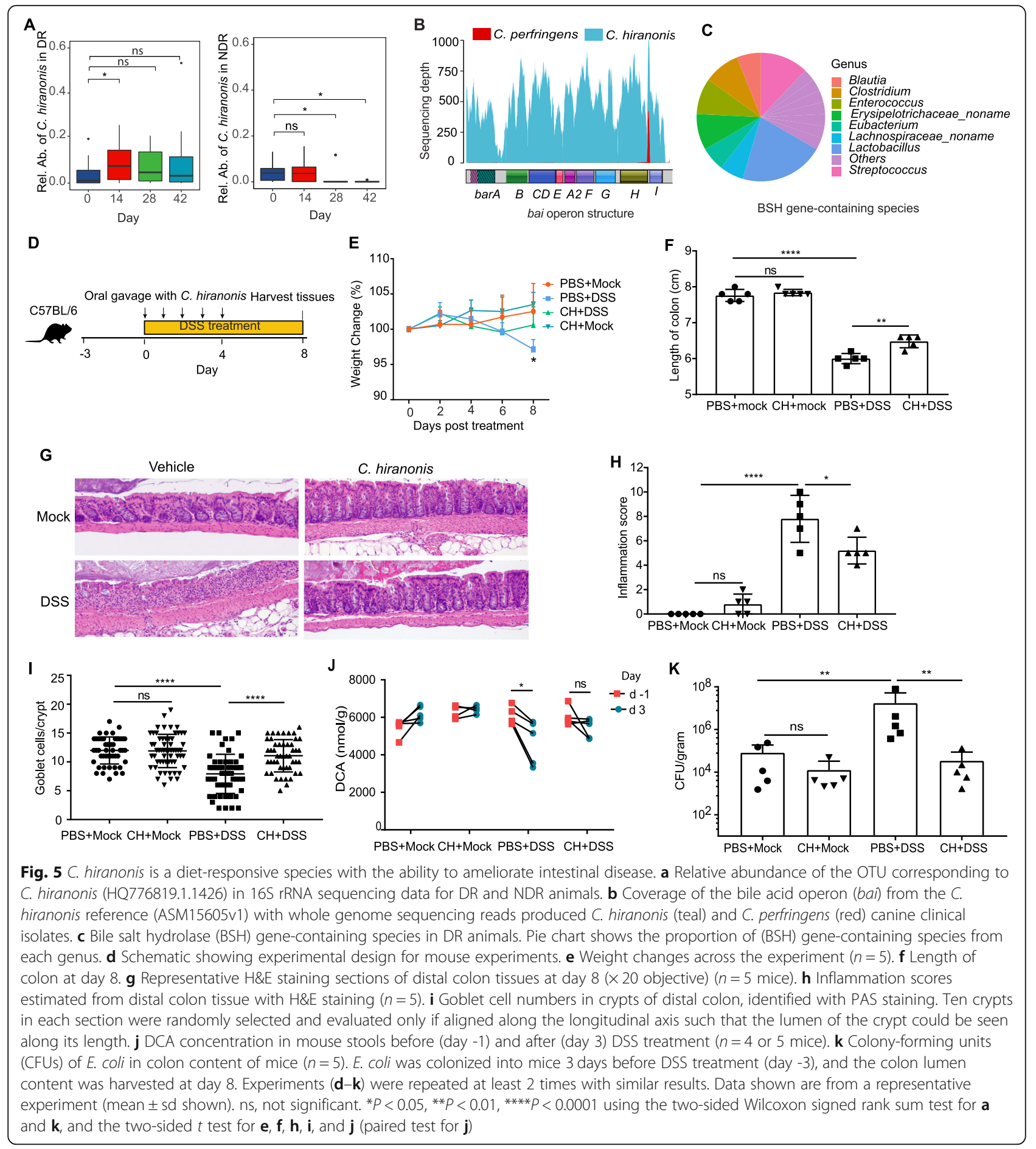

C. scindens was estimated using the proportion of total reads that map to the reference genome, revealing significant increases in this species and the bai operon from pretreatment to 8 weeks post-EEN (Fig. 6a, b, respectively). Remarkably, this increase was observed in patients that entered remission following EEN (responsive, $n=10$ ) but not those that failed therapy (non-responsive, $n=10$ )
(Fig. 6a, b). In addition, the correlation analysis between the relative abundance of $C$. scindens and fecal calprotectin (FCP), a biomarker of disease activity for IBD [23], indicated a significantly negative correlation (Fig. 6c) in diet-responsive patients $(R=-0.3515, P=0.03287)$, but not in non-responders $(R=-0.0267, P=0.877)$. Similarly, a significant negative correlation between bai operon and 
FCP was observed in diet-responsive $(R=-0.3944, P=$ $0.0157)$, but not non-responsive patients $(R=0.0490, P=$ 0.7766) (Fig. 6d). These results show that bile acid-producing clostridia are associated with diet-induced remission in both animals and humans alike (Fig. 6e).

\section{Discussion}

Using an animal model of food-responsive chronic inflammatory enteropathy, we showed that secondary bile acids and bile acid-producing Clostridium species ( $C$. hiranonis and $C$. scindens) are key features associated with diet-induced remission in humans and dogs. Previous studies reported dysregulated bile acid metabolism in IBD patients [51] and dogs with CE [52], and showed that $C$. hiranonis and E. coli were biomarkers of disease status in dogs [53]. Our results extend these observations by identifying C. hiranonis as a remission-associated taxon that may serve as a source of secondary bile acids. Furthermore, we observed that $C$. hiranonis preserved deoxycholic acid levels and ameliorated disease in DSS-treated mice, further highlighting a link between bile acid-producing clostridia and remission of gastrointestinal diseases. Our in vitro inhibition experiments with lithocholic and deoxycholic acids on the $C$. perfringens and $E$. coli isolates, together with previous findings of in vitro antibacterial activities of lithocholic acid on $E$. coli $[54,55]$, suggest that the increased C. hiranonis abundance could potentially contribute to the control of pathobionts in the gut. Indeed, as dogs entered remission following dietary therapy, we observed a remarkable decrease of $C$. perfringens and $E$. coli, coincident with increased levels of lithocholic and deoxycholic acid. In addition to being antimicrobial, physiological levels of these bile acids have been shown to modulate intestinal inflammation, in part due to their ability to signal through the farnesoid X receptor and other bile acid receptors to inhibit pro-inflammatory signaling [55-57]. Our data point to specific bile acids as being strongly associated with dietinduced remission, and warrant additional mechanistic studies to determine whether and how bile acid-producing clostridia modulate chronic inflammatory enteropathies in vivo.

Our findings complement recent studies showing that bile acid-producing clostridia mediate protection against $C$. difficile infection [58]. C. difficile infections frequently arise after antibiotic treatment, a phenomenon attributed to the effect of antibiotics on secondary bile acid levels [59]. Interestingly, we also observed that antibiotics antagonized the diet-induced shifts in microbiome composition and function, promoting a more dysbiotic state coincident with dramatically reduced levels of lithocholic and deoxycholic acid and elevated levels of $E$. coli (Figs. 2 and 3). Taken together with the antibacterial activities of secondary bile acids (Fig. 4), these data imply a possible general model for microbe-microbe interactions in the gut in which bile acid- producing clostridia may restrict the growth of a range of bile acid-sensitive pathobionts to limit disease, and highlight that these processes are exquisitely sensitive to antimicrobials. This model will need to be elaborated by in vitro and in vivo assays, since our data show that the extent to which bile-acid producers can restrict pathobiont growth will likely be dependent on the specific bile acid as well as the target microbe (Fig. 4). At physiologic levels, deoxycholic acid was more effective than lithocholic acid at restricting the growth of $E$. coli and $C$. perfringens. Yet, $E$. coli was notably more resistant, requiring concentrations of deoxycholic acid 10 times higher than what was needed to restrict $C$. perfringens. Several mechanisms mediating bile acid resistance have been described for $E$. coli, including the outer membrane acting as a barrier to the influx of bile salts [60], and activation of efflux pumps [61] and the SOS response [62]. However, little is known about the response of Clostridial species to bile acid stress, but differences in cell wall composition and physicochemical properties, such as hydrophobicity, between gram-negative and gram-positive bacteria may play a role. In addition, the observation of strain-specific variability in resistance to bile acids has been described for $C$. difficile [63], suggesting genetic factors may be involved. The poor solubility of many bile acids, together with evidence that supra-physiologic levels of bile acids are pathologic $[64,65]$, makes it challenging to directly manipulate the microbiome by administration of these compounds. However, our results showing that gavaging mice with $C$. hiranonis stabilize levels of deoxycholic acid during colitis suggest a possible indirect and safe way for manipulating intestinal bile acids. Whether or how $C$. hiranonis or secondary bile acids play a role in restricting E. coli, C. perfringens, or other pathobionts in vivo remains to be determined, but elucidating these mechanisms might have important health implications beyond veterinary medicine. Interestingly, when we examined data from a cohort of pediatric Crohn's disease patients before and after diet therapy, we found that $C$. scindens, a well-known secondary bile acid producer, was associated with diet-induced remission (Fig. 6). This is particularly relevant to our findings given a recent report that sustained remission following EEN was characterized by low levels of proteobacteria, while patients that relapse showed a marked increase in Proteobacteria [66]. Differences in the species of secondary bile acid producers observed between dogs and humans may be shaped by host-microbe interactions, but it is also possible that other bai operon- or BSH gene-containing species make important contributions to the secondary bile acid production and pathobiont restriction in vivo.

Rates of remission achieved with diet therapy in this and prior studies of canine CE [37] mirror remission rates achieved with EEN for treating pediatric Crohn's disease [17]. In both cases, however, a substantial portion of 


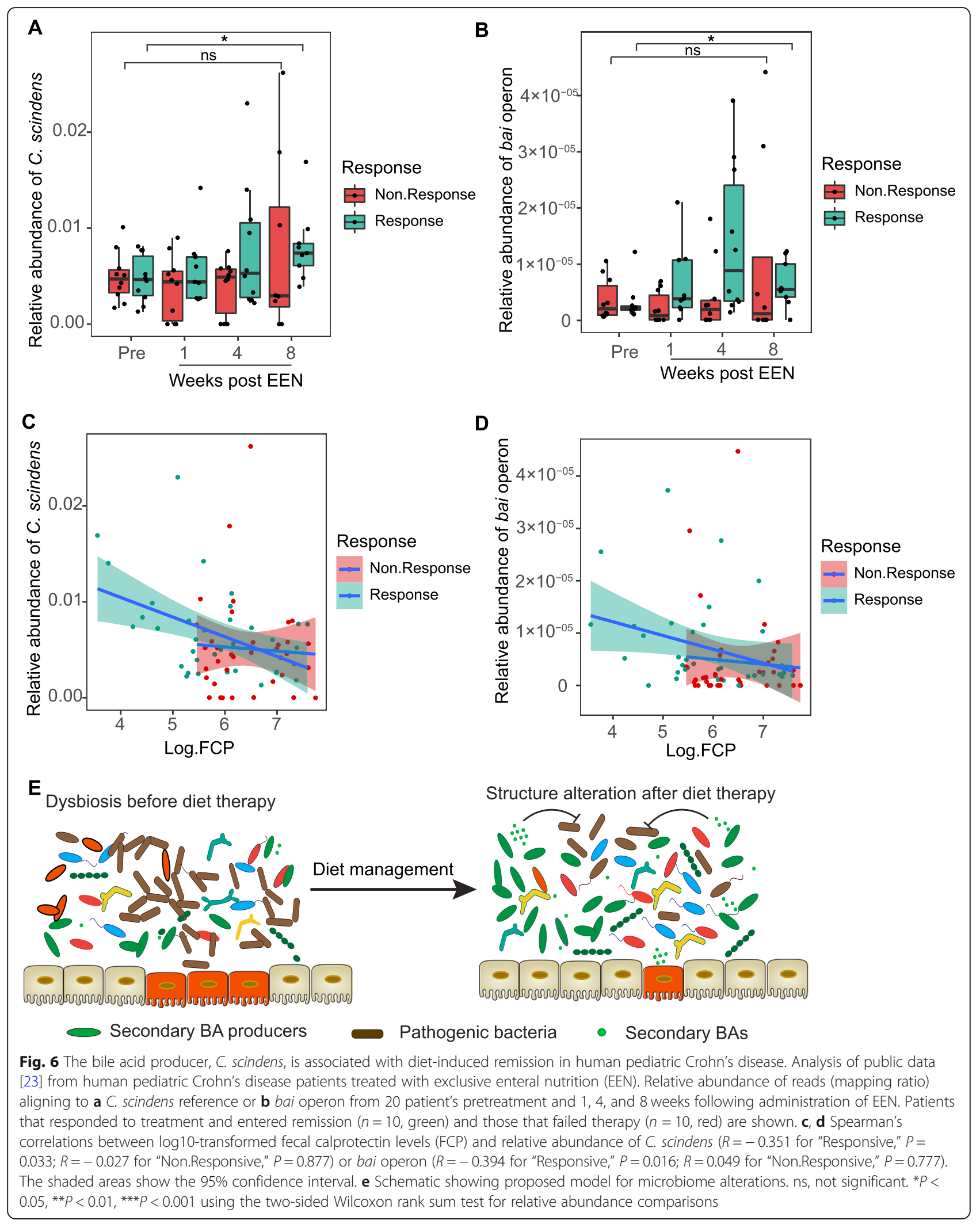


patients fail to respond to therapy. Understanding why some patients fail and what might be done to improve remission rates during diet therapy is critical in improving clinical care for inflammatory bowel diseases. Treatment outcome was not associated with differences in baseline levels of $C$. hiranonis, as both DR and NDR animals in our study had comparable levels of $C$. hiranonis prior to treatment, suggesting that microbe-microbe interactions, differing mucosal immune responses, or other factors may be necessary to support expansion of $C$. hiranonis during diet therapy. Compared to diet-responsive animals, those that failed treatment presented with higher overall disease scores. At first glance, this would seem to contradict evidence from human studies, where EEN achieved higher remission rates in children with a more severe disease (based on the Pediatric Crohn's Disease Activity Index, or PCDAI) and resulted in more mucosal healing (reduced fecal calprotectin levels) [17]. However, closer inspection of disease scores in our study (Additional file 1: Figure S11A) showed that NDR animals presented more frequently with vomiting and inappetence than their DR counterparts, indicating that these symptoms may limit the effectiveness of diet as therapy and raising the possibility that antiemetics and/or appetite stimulants could improve the efficacy of diet for some patients. The microbial and metabolic differences we observed between DR and NDR animals were still evident when only score-matched animals were considered in the analysis (Additional file 1: Figure S11), suggesting that the microbiome phenotype we have described is associated with treatment outcome rather than disease severity. Taken together, these data suggest that both clinical and microbial biomarkers could be used to predict patients that might be refractory to diet treatment.

The precise role that diet and dietary components play in remission has yet to be defined. One interpretation of our data is that therapeutic diet modifies the composition and/or function of the microbiome, which subsequently impacts pathobionts (e.g., E. coli and C. perfringens), host immunity, or both, thereby reducing pathology. Alternatively, diet may induce remission through mechanisms that are independent of the microbiome, in which case alterations to the microbiome and restoration of normal bile acid production would be consequences rather than causes of remission. Finally, diet may impact disease through both microbiome-dependent and microbiome-independent mechanisms. Nevertheless, given that certain bacteria and bile acids have been implicated in IBD pathogenesis and host inflammation [55], it seems unlikely that the reduced pathobionts and increased secondary bile acids have no contribution to remission in our model. Interestingly, a previous study showed that the composition of the gut microbiome was not affected when healthy dogs were fed a hydrolyzed diet [67]. Our data show that diet does, in fact, impact the microbiome of animals with disease, suggesting that therapeutic diets can have different effects depending on the basal state of the gut microbiome. Hydrolyzed protein diets, used in this study, have been shown to be effective in the management of canine CE [68, 69] and have previously been shown to be more effective for long-term management when compared to a highly digestible diet formulated with non-hydrolyzed protein sources $[68,69]$, but other studies have shown efficacy with nonhydrolyzed commercial prescription diets made from limited ingredients that include a novel protein source. Thus, one important open question is how therapeutic diets such as EEN or different prescription pet foods modulate disease and the microbiome and whether there are general principles that could be used to guide the development of better dietary interventions. Leveraging spontaneous animal models, such as the dog where formulated diets have long been a standard of care, will help dissect the therapeutic roles of different diets, thus providing valuable insight into human intestinal disease.

\section{Conclusions}

In summary, these data show that remission induced by a therapeutic hydrolyzed protein diet is linked to improved microbiota structure in canine chronic inflammatory enteropathy, marked by decreased relative abundance of pathobionts and increased abundance of a secondary bile acid producer (C. hiranonis). Levels of lithocholic and deoxycholic acid in stool rose rapidly after the diet therapy and inhibited the in vitro growth of disease-associated taxa. Mining public data from diet therapy in human pediatric Crohn's disease showed similar results, supporting a model by which dietary interventions lead to remission. These results warrant further investigations in other animal models and human studies and constitute an important first step in establishing a framework for evaluating the efficacy of dietary interventions, which could help guide the rational design of more effective therapeutic diets.

\section{Methods \\ Diagnosis and treatment of canine chronic enteropathy}

Client-owned animals presenting with clinical signs of canine chronic enteropathy (CCE) were screened at the Ryan Veterinary Hospital of the University of Pennsylvania for enrollment in a study to determine the Effect of Nutritional Therapy on Microbiome in Canine Enteropathy ("ENTiCE"). Dogs were screened if they had any one of the following clinical signs for $\geq 3$ weeks' duration: vomiting, diarrhea, or weight loss despite adequate caloric intake. Dogs were excluded from screening if they had been treated with a hydrolyzed protein diet, antibiotics, corticosteroids, or probiotics within the previous 2 weeks. At the time of screening, the following were performed on each animal: complete physical examination, routine fecal 
screening (including zinc sulfate flotation for parasite identification, gram stain and culture for Salmonella spp. and Campylobacter spp.), complete blood count, serum biochemical profile, serum measurement of canine trypsin-like immunoreactivity, cobalamin and folate, urinalysis, abdominal ultrasound examination, and disease severity scoring using the Canine Chronic Enteropathy Clinical Activity Index (CCECAI) [37]. If these initial screening tests failed to identify a cause for the clinical signs, upper and/or lower gastrointestinal endoscopy with mucosal biopsies was performed. Biopsies were fixed in formalin and embedded in paraffin, sections were stained with hematoxylin and eosin, and slides were examined by a board-certified veterinary pathologist. Dogs were enrolled only if histopathology revealed intestinal inflammation with no identifiable underlying cause (such as infectious agents). Dogs were excluded if another histopathologic diagnosis was identified.

The ENTiCE study included three 14-day treatment tiers (Fig. 1a and Additional file 1: Figure S1), and dogs were evaluated for a therapeutic response at the conclusion of each tier using CCECAI. Remission was determined using an abbreviated CCECAI that included scores to the first five indices (attitude/activity, appetite, vomiting, stool consistency, and stool frequency), and was defined as an abbreviated CCECAI score $\leq 2$, with no score $>1$ for any of the five indices. Animals were first administered a therapeutic hydrolyzed protein diet (Royal Canin Veterinary diet Hypoallergenic Adult dry dog). Dogs that entered remission following this treatment were designated as diet-response (DR) and were maintained on therapeutic diet for the reminder of the trial. Animals that did not respond to therapeutic diet (NDR) subsequently began a 2-week course of metronidazole $(10 \mathrm{mg} / \mathrm{kg}$ PO q $12 \mathrm{~h})$ while being maintained on the therapeutic diet. Dogs that entered remission following antibiotic treatment were maintained on the combination of antibiotics and therapeutic diet for the reminder of the trial. Animals that still failed to show a favorable response remained on diet and metronidazole, but received prednisone $(1 \mathrm{mg} / \mathrm{kg} \mathrm{PO} \mathrm{q}$ $12 \mathrm{~h}$ ) (tier 3) for the final 14 days of the trial. Dogs that presented with hypoalbuminemia (protein-losing enteropathy) at the initial screening were presumed to have more severe disease and poorer prognoses and thus were immediately administered all three interventions, and were not included in our analyses. All dogs in which serum cobalamin was low at screening were supplemented with cyanocobalamin $(50 \mathrm{mcg} / \mathrm{kg}$ SQ q 7 days) for the duration of the study. At the conclusion of the study, all animals returned to the clinic for the primary endpoint, which included a full re-evaluation of dogs including complete physical examination, complete blood count, serum chemistry, serum measurement of cobalamin and folate (if low at screening visit), urinalysis, CCECAI scoring, and final fecal collections.

\section{S rRNA gene sequencing and data analysis}

Genomic DNA was extracted from stool using the PowerSoil DNA Isolation Kit (MO BIO Laboratories, Carlsbad, CA) following the manufacturer's recommendations. A mock community pool containing purified genomic DNA from 12 known bacterial isolates was amplified and sequenced as a quality control. Additional controls included extraction of blank-processed samples (in which the DNA extraction process was followed without addition of input material), and water only, to determine background microbial signal. A dual-index amplicon sequencing method was employed for PCR amplification of the V4 region of the 16S rRNA gene [70]. Pico-green-based amplicons were sequenced on a MiSeq platform (Illumina, San Diego, CA) using $250 \mathrm{bp}$ paired-end chemistry. Reads were filtered to remove sequences with average Phred quality score $\leq$ 20 using Quantitative Insights into Microbial Ecology (QIIME) [71] with filtering options (-q 20 -p 0.75 -r 3). Homopolymers $>10 \mathrm{bp}$ in length and sequences $<250 \mathrm{bp}$ and $>256$ bp were removed using Mothur [72]. Chimeric sequences were identified and removed by usearch61 [73] against the representative $16 \mathrm{~S}$ sequences of SILVA128 (97_ otus_16S.fasta) [74, 75]. Quality-controlled sequences were then clustered against the SILVA128 database (SILVA 128_QIIME_release) using the open-reference OTU picking as implemented in QIIME with default parameters. Analysis of OTU tables was carried out using the R statistical environment [76], the bioconductor suite of software [77], and the Phyloseq2 package [78]. Singletons and OTUs with ambiguous annotations were removed from the OTU table. The OTU table was rarefied to 10,600 sequences per sample. Alpha diversity (Shannon's diversity index and Faith's phylogenetic diversity) and beta diversity (weighted and unweighted UniFrac) were calculated using Phyloseq2. Pielou's evenness index was calculated as previously described [79].

For differential abundance analysis and association analysis, filtering on un-rarefied OTU table of samples with > 10,000 reads was carried out to remove taxa with a max abundance $<0.1 \%$ across all samples and present in $<10 \%$ of all samples. DESeq2 [80] implemented in Phyloseq2 (test $=$ "Wald," fitType = "parametric") was used for differential abundance analysis on different taxonomy levels (fold change $>2$ and $P$ value $<0.05$ ) using un-rarefied reads. To estimate the effect from outliers, separate fold changes of relative abundances ( 1 read was added to avoid taking log of zero) were also calculated by excluding the $10 \%$ outliers (5th-95th) in each group. The Spearman correlation was computed between the abundance of each microbial composition log-transformed) and the values of different factors (i.e., CCECAI for each dog, time points, concentration of each metabolite). To avoid taking log of the zero value, we added 1 read to the abundance for each composition before calculating the Spearman correlation. 
All $P$ values in the above analysis were adjusted by the FDR (Benjamini-Hochberg) method for multiple comparisons except where noted. In order to get a taxonomic assignment at species level for the OTUs from Clostridium sensu stricto 1 , the corresponding representative sequences in SILVA database were used to search against NCBI "nr" database. Species were temporarily assigned by the best hits $(P<1 \mathrm{e}-5)$, and further confirmations were done by comparing the relative abundances of these species determined by metagenomic shotgun sequencing method and by $16 \mathrm{~S}$ sequencing method. The OTU "New.ReferenceOTU131" represents C. perfringens, which is the most dominant OTU in some dogs, and OTU "HQ776819.1.1426" corresponds to C. hiranonis.

\section{Metagenomic sequencing and data analysis}

Sequencing libraries were prepared using Illumina Nextera XT with 1 ng DNA extracted from canine stool collected at days 0,14 , and 42 from 18 out of the 20 diet-responsive dogs in the study. Sizing and quantification of libraries were carried out using a Tapestation 4200 (Agilent) and Qubit 3 (Thermo Fisher), respectively. Equimolar amounts of each library were pooled and sequenced on an Illumina NextSeq 500 instrument to produce $150 \mathrm{bp}$ paired-end sequences. Sequencing adapters and low-quality reads were trimmed and filtered by Trimmomatic (v0.36) (leading:3 trailing: 3 slidingwindow:4:15 minlen:36). High-quality reads were mapped to the canine reference genome (CanFam3.1), using Bowtie2 v2.3.4.1 (--very-sensitive), and aligned reads were removed using SamTools [81]. After host read filtering, each sample had a depth of at least 10 million paired-end reads (median depth $=35.8$ million). Taxonomic annotation of each sample was generated using Metaphlan2 [49]. Functional and pathway composition was calculated with HUMAnN2 [82] using the UniRef50 database and UniPathway database with default settings. The abundance of each gene family in each species was estimated using HUMAnN2 and normalized with relative abundance. Bile salt hydrolase/choloylglycine hydrolase (BSH)-coding genes and genes in bai operon in microbiome were identified from the gene family search result of HUMAnN2 according the reference cluster IDs of these genes in UniRef50. Low abundance gene pathways with max abundance $<0.01 \%$ across all samples or present in $<$ $25 \%$ samples were filtered out. Two-sided Wilcoxon signed-rank test was used for comparisons of UniPathway pathways at different timepoints $(F D R<0.05)$. Principal component analysis for pathways was performed by the $\mathrm{R}$ package factoextra. The identified Clostridium spp. and Eubacterium spp. were further searched for the existence of genes involved in secondary bile acid production (bai operon) using tBlastn against the reference genomes of these species in GenBank with the protein sequence of genes in $7 \alpha$-dehydroxylation pathway (baiG, baiB, baiA, baiF, baiCD, and baiE) ( $P$ value $\leq 1 \mathrm{e}-5)$.

Metagenomic data from pediatric Crohn's disease patients before and after exclusive enteral nutrition (EEN) have been described previously [23] and were downloaded from European Nucleotide Archive (ENA) (SRP057027). The same filtering steps and settings for the metagenomic data analysis above in this study were used for these datasets. After filtering out human reads, taxonomic annotation for each sample using Metaphlan2 showed the presence of Clostridium. Among them, Clostridium scindens has been well known for the secondary BA-producing ability. Paired reads with PCR duplicates removed by samtools [81] were aligned to the C. scindens reference genome (ASM15450v1, strain ATCC 35704) as well as strain VE202-05 (ASM47184v1) using bwa-mem (v0.7.17r1188) [83] with default settings to estimate the relative abundances of bacteria among different samples (proportion of mapped reads in total reads). Wilcoxon's sum rank test was used to test for significant differences in read mapping, and Spearman's correlation was used to compare number of reads mapped with log-transformed fecal calprotectin (FCP) levels [23].

\section{Anaerobic culture and identification of bacterial isolates by whole genome sequencing}

Rectal swabs freshly collected from dogs with active disease (day 0 ) and/or in remission at the end of the study (day 42) were transferred to an anaerobic chamber $(97.5 \%$ nitrogen, 2.5\% hydrogen; Coy Labs, Grass Lake, MI) within $1 \mathrm{~h}$ of collection. The tip of each swab was homogenized in $1 \mathrm{~mL}$ of pre-reduced PBS with $1 \%$ cysteine (PBSc). Serial dilutions made in PBSc (down to $10^{-5}$ ) were plated on brain-heart infusion (BHI), yeast casitone fatty acid with carbohydrate (YCFAC) [84], gut microbiota medium (GMM) [85], and De Man, Rogosa, and Sharpe (MRS) [86] agars (Anaerobe Systems, Morgan Hill, CA). After incubation at $37^{\circ} \mathrm{C}$ for $1-3$ days, single colonies were picked from plates and grown overnight in BHI, YCFAC, GMM, or MRS broth (Anaerobe Systems, Morgan Hill, CA). Overnight cultures were saved as glycerol stocks (25\% glycerol) and frozen neat for DNA extraction. DNA was purified from bacterial isolates using the High Pure PCR template kit (Roche) and used for PCR with primers specific for the bacterial 16S rRNA gene, including 27F (5'-AGAGTTTGATCMTGGCTCAG-3'), 515F (5'-GTGCCAGCMGCCGCGGTAA-3'), and 1492R (5'CGGTTACCTTGTTACGACTT-3'). PCR products were purified using QiaQuick PCR Purification kit (Qiagen) and Sanger sequenced, and sequences were assembled using Geneious software v11.1.5 (Biomatters, Inc.). The longest high-quality stretch of assembled sequence (at least $800 \mathrm{bp}$ ) was used for BLAST to find the closest match in GenBank. In addition, for selected C. hiranonis, $C$. 
perfringens, and E. coli isolates, $1 \mathrm{ng}$ of DNA was used to construct sequencing libraries using Illumina Nextera XT. Libraries were sized and quantified as described above for metagenomic sequencing. Sourmash was used to detect contamination reads from metagenomic sequencing for the isolates [87]. For each sample, at least $\sim 10$ million, $150 \mathrm{bp}$ single-end reads were generated using an Illumina NextSeq 500 instrument. Quality control steps were the same as the metagenomic analysis above. High-quality reads were mapped to the genome of C. hiranonis (ASM15605v1) using Stampy [88] (--substitutionrate $=0.1$, which allows mapping of reads that are highly divergent from the reference genome. PCR duplicates were removed by Samtools. Coverage of genomic regions representing the bai operon was calculated for each isolate to show the existence of genes in $7 \alpha$-dehydroxylation pathway.

\section{Metabolomics and in vitro bacterial growth inhibition assays}

Bile acids were quantified in stool using a Waters Acquity uPLC System with a QDa single quadrupole mass detector and an autosampler as described previously [89]. Briefly, fecal samples were suspended in methanol $(5 \mu \mathrm{L} / \mathrm{mg}$ stool), vortexed for $1 \mathrm{~min}$, and centrifuged at $13,000 \mathrm{~g}$ for $5 \mathrm{~min}$. The supernatant was transferred to a new vial and analyzed on an Acquity uPLC with a Cortecs UPLC C$18+1.6 \mathrm{~mm} 2.1 \times 50 \mathrm{~mm}$ column. All chemicals and reagents were mass spectrometry grade. The differential level analysis of bile acids was performed using the twosided Wilcoxon signed-rank test. For the component below detection limit $(<1 \mathrm{nmol} / \mathrm{g}$ ), the value 0.5 (half the limit of detection) was given for analysis. Canine isolates of $C$. perfringens $(n=3)$ and $E$. coli were revived from glycerol stocks in Modified Reinforced Clostridial Broth (MRCB, Fisher Scientific) or Luria broth (LB, Fisher Scientific), respectively, and grown overnight in the anaerobic chamber at $37^{\circ} \mathrm{C}$. Lithocholic and deoxycholic acids (Sigma) were dissolved in $100 \%$ ethanol $(30 \mathrm{mg} / \mathrm{mL})$. Growth inhibition by deoxycholic acid was determined by microbroth dilution and assessed by $\mathrm{OD}_{630}$ after overnight growth. Due to low solubility $(<1 \mathrm{mg} / \mathrm{L})$, inhibition by lithocholic acid was assessed by counting colonies on agar plates with LCA $(0,0.1,0.25,0.5,0.75$, or $1 \mathrm{mg} / \mathrm{mL}$ and LB plates for E. coli, and 0, 0.01, 0.025, 0.05, 0.075, 0.01, $0.25,0.50,0.75$, or $1 \mathrm{mg} / \mathrm{mL}$ and Columbia blood agar supplemented with $5 \%$ defibrinated sheep's blood for $C$. perfringens) that were incubated anaerobically, at $37^{\circ} \mathrm{C}$ for 24 (E. coli) or $48 \mathrm{~h}$ (C. perfringens). Growth curves (Fig. 4c, d) were run in 96-well plates using an Epoch2 plate reader (BioTek, Vermont, USA). Optical density measurements were taken at $600 \mathrm{~nm}$ every $15 \mathrm{~min}$ for $24 \mathrm{~h}$. Wells containing $200 \mu \mathrm{L}$ of the appropriate medium and bile acid levels were inoculated 1:100 (v/v) from standing overnight cultures. Technical replicates $(n=3)$ were run for bacterial strain under each condition. All procedures were conducted in an anaerobic chamber (Coy Labs, Michigan, USA).

\section{Mouse experiments}

Female 7-week-old C57BL/6 J mice (specific pathogen-free) were purchased from Jackson Laboratories and maintained for 2-3 weeks in the animal facility at University of Pennsylvania prior to the to the start of dextran sulfate sodium (DSS) treatment. Animals were randomly assigned to groups ( $n=5$ mice per cage) at baseline, and drinking water was replaced with either filter-sterilized water (mock-treatment) or a filter-sterilized solution of $2.5 \%(\mathrm{w} /$ v) DSS (relative molecular mass $\sim 40,000$; Sigma-Aldrich) in water. The mice treated with mock or DSS were orally gavaged $C$. hiranonis $\left(1 \times 10^{8} \mathrm{CFU} / \mathrm{mouse}\right.$, in anaerobic PBS) or anaerobic PBS (control) from days 0 to 4 . C. hiranonis was grown overnight in MRCB, anaerobically, at $37^{\circ} \mathrm{C}$. Culture density was assessed via optical density $(630 \mathrm{~nm})$ and the required volume of culture was spun at $10,000 \mathrm{~g}$ for $15 \mathrm{~min}$. The consumption of DSS water was monitored every day, and no significant difference was observed between the groups. Stool samples were collected at day -1 and day 3 for metabolomic analysis. Mice were euthanized on day 8 , and colon tissues were collected. Distal colons were cut, fixed in formalin, embedded in paraffin, and stained with hematoxylin and eosin (H\&E) or Periodic acid-Schiff (PAS). Histologic sections were evaluated by a board-certified veterinary pathologist (CB). Colitis was scored according to previously published criteria [90, 91]. Briefly, points were assigned to degree of mucosal/crypt loss (0: normal to 4: loss of all epithelium), lamina propria mononuclear infiltrate (0: normal to 3: marked increase), neutrophilic infiltrate ( 0 : normal to 3: marked increase), epithelial hyperplasia (0: normal to 3 : marked), and edema (0: normal to 3 : severe and transmural).

For E. coli colonization experiments (Fig. 5k), C57BL/6 J mice were orally gavaged with kanamycin-resistant $E$. coli Nissle strain $1917\left(1 \times 10^{9} \mathrm{CFU} /\right.$ mouse $)$ in LB media at day -3 . Then, the mice were subjected to the same procedure as described above (treated with either DSS or mock; gavaged with $C$. hiranonis or anaerobic PBS). At day 8, colonic content was harvested, weighed, and plated on LB agar plates under kanamycin selection $(100 \mu \mathrm{g} / \mathrm{mL})$. E. coli colonies were counted and normalized by sample weight for each mouse.

\section{Additional files}

Additional file 1: Figure S1. Detailed clinical design of the 'ENTiCE' canine chronic enteropathy study. Figure S2. Community structure of microbiomes in the dogs with CE and in the healthy dogs. Figure S3. Differentially abundant OTUs between healthy animals and CE animals. 
Figure S4. Microbiota community structure changes induced by diet therapy. Figure S5. OTU level (mean values) dynamics of microbiota structure for DR animals throughout the study. Figure S6.

Concentrations of bile acids detected in fecal samples from dietresponsive dogs. Figure S7. Bile salt hydrolase (BSH) abundance in DR animals. Figure S8. Heatmap of the top 25 most abundant species across the samples in DR animals based on metagenomic data. Figure S9. Relative abundance of $C$. hiranonis in diet responsive dogs calculated from metagenomic data. Figure S10. Representative H\&E staining sections of distal colon tissues at day 8 (40 x objective). Figure S11. Disease score-matched analysis. (PDF $5033 \mathrm{~kb}$ )

Additional file 2: Table S1. OTUs with differential abundances between samples of healthy dogs and dogs with CE at day 0 (Healthy vs CE day 0). Table S2. OTUs with differential abundances between day 0-samples of diet responsive dogs and non-diet responsive dogs. Table S3. Genera with differential abundances between samples of day 14 and day 0 (day 14 versus day 0) for diet responsive dogs. Table S4. OTUs with differential abundances between samples of day 14 and day 0 for diet responsive dogs (day 14 versus day 0). Table S5. OTUs with differential abundances between samples of day 14 and day 0 (day 14 versus day 0 ) for non-diet responsive dogs. Table S6. Differentially abundant pathways between day 14 and day 0 after diet treatment from metagenomic analysis for diet-responsive dogs (fold-change $>1.5$ and $P$-value $<0.05$ ). Table S7. Comparisons of bile acids in samples at different timepoints for diet responsive dogs. Table S8. Spearman correlations between abundance of OTUs and concentration of Bile acids in diet responsive dogs. Table S9. Spearman correlations between bile acids and bile salt hydolases (BSHs) in diet-responsive dogs $(P<0.05)$. (XLSX $135 \mathrm{~kb})$

\section{Abbreviations}

ENTiCE: Effect of Nutritional Therapy on Microbiome in Canine Enteropathy; PLEASE: Pediatric Longitudinal Study of Elemental Diet and Stool Microbiome Composition; BA: Bile acid; BSH: Bile salt hydrolase; CCE: Canine chronic enteropathy; CCECAl: Canine Chronic Enteropathy Clinical Activity Index; CE: Chronic enteropathy; DCA: Deoxycholic acid; DR: Diet-responsive; EEN: Exclusive enteral nutrition; ENA: European Nucleotide Archive: FDR: False discovery ratio; FMT: Fecal microbiota transplant; FRE: Foodresponsive enteropathy; IBD: Human inflammatory bowel disease; LCA: Lithocholic acid; NDR: Non-diet-responsive; OTU: Operational taxonomic unit; PEN: Partial enteral nutrition; QIIME: Quantitative Insights into Microbial Ecology; SRA: Sequence Read Archive

\section{Acknowledgements}

We thank Stephen Cole and Kathleen O'Shea for assistance with bacterial growth and helpful discussions, and Sebastian Winter (UT Southwestern) for generously providing E. coli Nissle 1917. We thank Michael DiGregorio and the Veterinary Clinical Investigations Center (VCIC) at PennVet for support and consultation in study design and execution, and Dr. Gary Wu (University of Pennsylvania) for critical feedback during manuscript preparation. We thank Royal Canin International for supporting the canine clinical trial. We thank all the clients and their dogs who dedicated their time to this study.

\section{Authors' contributions}

DPB, CAH, SW, and ESF designed the experiments. MPR and RE planned and ran the canine clinical study and collected the samples. DPB, SW, MCS, ESF, $A M M, A E, E C P D M, K O, Y C$, and $G Z$ performed the experiments. $C B$ analyzed the histopathology for canine and murine samples. RNB advised on the inclusion and analysis of pediatric Crohn's disease data. DPB and SW analyzed the data. DPB, SW, and ESF wrote the manuscript, with input from all co-authors. All authors read and approved the final manuscript.

\section{Funding}

DPB was partially supported by a grant from the Margaret Q Landenberger Family Research Foundation. ECPDM is grateful to São Paulo Research Foundation, Brazil (Grant\#2016/16293-5). A Tobacco Formula grant provided partial support for the project and for RNB. This Tobacco Formula grant is under the Commonwealth Universal Research Enhancement (CURE) program with the grant number SAP \# 4100068710. The funders had no role in data collection and analysis, decision to publish, or preparation of the manuscript.

\section{Availability of data and materials}

Raw 165 rRNA gene sequences and metagenomic sequences for all samples used in this study have been deposited in the Sequence Read Archive (SRA) under project accession no. PRJNA515316. Processed 165 data and clinical metadata for the ENTiCE study are also available on microbiomeDB.org [92]. Original R scripts, metadata, and OTU table are available on Github at https://github.com/dpbisme/Wang_ENTiCE_study, and have been archived on Zenodo (DOl: https://doi.org/10.5281/zenodo.3379096).

\section{Ethics approval and consent to participate}

All animal work was carried out in accordance within the guidelines of the University of Pennsylvania IACUC (protocol 805283 for canine, and 805045 for mouse). Signed owner consent was obtained before enrollment of dogs in the ENTiCE study.

\section{Consent for publication}

Not applicable.

\section{Competing interests}

The authors declare that they have no competing interests.

\section{Author details}

'Department of Pathobiology, School of Veterinary Medicine, University of Pennsylvania, Philadelphia, PA 19104, USA. ${ }^{2}$ Department of Clinical Sciences and Advanced Medicine, School of Veterinary Medicine, University of Pennsylvania, Philadelphia, PA 19104, USA. ${ }^{3}$ Division of Gastroenterology, School of Medicine, University of Pennsylvania, Philadelphia, PA 19104, USA. ${ }^{4}$ Faculdade de Ciências Farmacêuticas de Ribeirão Preto, Universidade de São Paulo, Ribeirão Preto, SP, Brazil. ${ }^{5}$ Department of Pediatric Gastroenterology Hepatology and Nutrition, Children's Hospital of Philadelphia, Philadelphia, PA 19104, USA.

Received: 14 March 2019 Accepted: 19 August 2019

Published online: 31 August 2019

\section{References}

1. Nitzan O, Elias M, Peretz A, Saliba W. Role of antibiotics for treatment of inflammatory bowel disease. World J Gastroenterol. 2016;22:1078-87. https://doi.org/10.3748/wjg.v22.i3.1078.

2. Khan KJ, Ullman TA, Ford AC, Abreu MT, Abadir A, Abadir A, et al. Antibiotic therapy in inflammatory bowel disease: a systematic review and meta-analysis. Am J Gastroenterol. 2011;106:661-73. https://doi.org/10.1038/ajg.2011.72.

3. Knights D, Lassen KG, Xavier RJ. Advances in inflammatory bowel disease pathogenesis: linking host genetics and the microbiome. Gut. 2013;62:150510. https://doi.org/10.1136/gutjnl-2012-303954.

4. Garrett WS, Lord GM, Punit S, Lugo-Villarino G, Mazmanian SK, Ito S, et al. Communicable ulcerative colitis induced by T-bet deficiency in the innate immune system. Cell. 2007:131:33-45. https://doi.org/10.1016/j.cell.2007.08.017.

5. Couturier-Maillard A, Secher T, Rehman A, Normand S, De Arcangelis A, Haesler R, et al. NOD2-mediated dysbiosis predisposes mice to transmissible colitis and colorectal cancer. J Clin Invest. 2013;123:700-11. https://doi.org/1 $0.1172 / \mathrm{JCl} 162236$.

6. Moon C, Baldridge MT, Wallace MA, D C-A, Burnham, Virgin HW, et al. Vertically transmitted faecal lgA levels determine extra-chromosomal phenotypic variation. Nature. 2015;521:90-3. https://doi.org/10.1038/ nature14139.

7. David LA, Maurice CF, Carmody RN, Gootenberg DB, Button JE, Wolfe BE, et al. Diet rapidly and reproducibly alters the human gut microbiome. Nature. 2014:505:559-63. https://doi.org/10.1038/nature12820.

8. Muegge BD, Kuczynski J, Knights D, Clemente JC, González A, Fontana L, et al. Diet drives convergence in gut microbiome functions across mammalian phylogeny and within humans. Science. 2011;332:970-4. https://doi.org/1 $0.1126 /$ science.1198719.

9. Lee D, Albenberg L, Compher C, Baldassano R, Piccoli D, Lewis JD, et al. Diet in the pathogenesis and treatment of inflammatory bowel diseases. Gastroenterology. 2015;148:1087-106. https://doi.org/10.1053/j.gastro.2015.01.007.

10. Levine A, Sigall Boneh R, Wine E. Evolving role of diet in the pathogenesis and treatment of inflammatory bowel diseases. Gut. 2018;67:1726-38. https://doi.org/10.1136/gutjnl-2017-315866.

11. Chassaing B, Koren O, Goodrich JK, Poole AC, Srinivasan S, Ley RE, et al. Dietary emulsifiers impact the mouse gut microbiota promoting colitis and 
metabolic syndrome. Nature. 2015;519:92-6. https://doi.org/10.1038/ nature14232.

12. Desai MS, Seekatz AM, Koropatkin NM, Kamada N, Hickey CA, Wolter M, et al. A dietary fiber-deprived gut microbiota degrades the colonic mucus barrier and enhances pathogen susceptibility. Cell. 2016;167:1339-53.e21. https://doi.org/10.1016/j.cell.2016.10.043.

13. Hou JK, Abraham B, El-Serag H. Dietary intake and risk of developing inflammatory bowel disease: a systematic review of the literature. Am J Gastroenterol. 2011;106:563-73. https://doi.org/10.1038/ajg.2011.44.

14. Ruemmele FM, Veres $\mathrm{G}$, Kolho KL, Griffiths A, Levine A, Escher JC, et al. Consensus guidelines of ECCO/ESPGHAN on the medical management of pediatric Crohn's disease. J Crohns Colitis. 2014;8:1179-207. https://doi.org/1 0.1016/j.crohns.2014.04.005

15. Cohen-Dolev N, Sladek M, Hussey S, Turner D, Veres G, Koletzko S, et al. Differences in outcomes over time with exclusive enteral nutrition compared with steroids in children with mild to moderate crohn's disease: results from the GROWTH CD study. J Crohns Colitis. 2018;12:306-12. https://doi.org/10.1093/ecco-jcc/jjx150.

16. Sigall-Boneh R, Pfeffer-Gik T, Segal I, Zangen T, Boaz M, Levine A. Partial enteral nutrition with a Crohn's disease exclusion diet is effective for induction of remission in children and young adults with Crohn's disease. Inflamm Bowel Dis. 2014;20:1353-60. https://doi.org/10.1097/ MIB.0000000000000110

17. Lee D, Baldassano RN, Otley AR, Albenberg L, Griffiths AM, Compher C, et al. Comparative effectiveness of nutritional and biological therapy in North American children with active crohn's disease. Inflamm Bowel Dis. 2015;21: 1786-93. https://doi.org/10.1097/MIB.0000000000000426.

18. Borrelli O, Cordischi L, Cirulli M, Paganelli M, Labalestra V, Uccini S, et al. Polymeric diet alone versus corticosteroids in the treatment of active pediatric Crohn's disease: a randomized controlled open-label trial. Clin Gastroenterol Hepatol. 2006;4:744-53. https://doi.org/10.1016/j.cgh.2006. 03.010.

19. Kaakoush NO, Day AS, Leach ST, Lemberg DA, Nielsen S, Mitchell HM. Effect of exclusive enteral nutrition on the microbiota of children with newly diagnosed Crohn's disease. Clin Transl Gastroenterol. 2015;6:e71. https://doi. org/10.1038/ctg.2014.21

20. Quince C, ljaz UZ, Loman N, Eren AM, Saulnier D, Russell J, et al. Extensive modulation of the fecal metagenome in children with crohn's disease during exclusive enteral nutrition. Am J Gastroenterol. 2015;110:1718-29; quiz 1730. https://doi.org/10.1038/ajg.2015.357.

21. Gerasimidis K, Bertz M, Hanske L, Junick J, Biskou O, Aguilera M, et al. Decline in presumptively protective gut bacterial species and metabolites are paradoxically associated with disease improvement in pediatric Crohn's disease during enteral nutrition. Inflamm Bowel Dis. 2014;20:861-71. https:// doi.org/10.1097/MIB.0000000000000023.

22. Schwerd T, Frivolt K, Clavel T, Lagkouvardos I, Katona G, Mayr D, et al. Exclusive enteral nutrition in active pediatric Crohn disease: effects on intestinal microbiota and immune regulation. J Allergy Clin Immunol. 2016; 138:592-6. https://doi.org/10.1016/j.jaci.2015.12.1331.

23. Lewis JD, Chen EZ, Baldassano RN, Otley AR, Griffiths AM, Lee D, et al. Inflammation, antibiotics, and diet as environmental stressors of the gut microbiome in pediatric crohn's disease. Cell Host Microbe. 2015;18:489500. https://doi.org/10.1016/j.chom.2015.09.008.

24. D'Argenio V, Precone V, Casaburi G, Miele E, Martinelli M, Staiano A, et al. An altered gut microbiome profile in a child affected by crohn's disease normalized after nutritional therapy. Am J Gastroenterol. 2013;108:851-2. https://doi.org/10.1038/ajg.2013.46.

25. Wu GD, Chen J, Hoffmann C, Bittinger K, Chen Y-Y, Keilbaugh SA, et al Linking long-term dietary patterns with gut microbial enterotypes. Science. 2011;334:105-8. https://doi.org/10.1126/science.1208344.

26. Gurry T, HST Microbiome Consortium*, Gibbons SM, LTT N, Kearney SM, Ananthakrishnan $\mathrm{A}$, et al. Predictability and persistence of prebiotic dietary supplementation in a healthy human cohort. Sci Rep. 2018;8:12699. https:// doi.org/10.1038/s41598-018-30783-1.

27. Wu GD, Compher C, Chen EZ, Smith SA, Shah RD, Bittinger K, et al. Comparative metabolomics in vegans and omnivores reveal constraints on diet-dependent gut microbiota metabolite production. Gut. 2016;65:63-72. https://doi.org/10.1136/gutjnl-2014-308209.

28. Obregon-Tito AJ, Tito RY, Metcalf J, Sankaranarayanan K, Clemente JC, Ursell LK, et al. Subsistence strategies in traditional societies distinguish gut microbiomes. Nat Commun. 2015;6:6505. https://doi.org/10.1038/ncomms7505.
29. Smits SA, Leach J, Sonnenburg ED, Gonzalez CG, Lichtman JS, Reid G, et al. Seasonal cycling in the gut microbiome of the Hadza hunter-gatherers of Tanzania. Science. 2017;357:802-6. https://doi.org/10.1126/science.aan4834.

30. Cerquetella M, Spaterna A, Laus F, Tesei B, Rossi G, Antonelli E, et al. Inflammatory bowel disease in the dog: differences and similarities with humans. World J Gastroenterol. 2010;16:1050-6.

31. Jergens $A E$, Simpson KW. Inflammatory bowel disease in veterinary medicine. Front Biosci (Elite Ed). 2012:4:1404-19.

32. Peiravan A, Bertolini F, Rothschild MF, Simpson KW, Jergens AE, Allenspach $\mathrm{K}$, et al. Genome-wide association studies of inflammatory bowel disease in German shepherd dogs. PLoS ONE. 2018;13:e0200685. https://doi.org/10.13 71/journal.pone.0200685.

33. Vázquez-Baeza Y, Hyde ER, Suchodolski JS, Knight R. Dog and human inflammatory bowel disease rely on overlapping yet distinct dysbiosis networks. Nat Microbiol. 2016;1:16177. https://doi.org/10.1038/nmicrobiol.2016.177.

34. Suchodolski JS, Dowd SE, Wilke V, Steiner JM, Jergens AE. 16 S rRNA gene pyrosequencing reveals bacterial dysbiosis in the duodenum of dogs with idiopathic inflammatory bowel disease. PLoS ONE. 2012;7:e39333. https:// doi.org/10.1371/journal.pone.0039333.

35. Simpson KW, Dogan B, Rishniw M, Goldstein RE, Klaessig S, McDonough PL, et al. Adherent and invasive Escherichia coli is associated with granulomatous colitis in boxer dogs. Infect Immun. 2006;74:4778-92. https://doi.org/10.1128/IAl.00067-06.

36. Coelho LP, Kultima JR, Costea PI, Fournier C, Pan Y, Czarnecki-Maulden G, et al. Similarity of the dog and human gut microbiomes in gene content and response to diet. Microbiome. 2018;6:72. https://doi.org/10.1186/s40168-018-0450-3.

37. Allenspach K, Wieland B, Gröne A, Gaschen F. Chronic enteropathies in dogs: evaluation of risk factors for negative outcome. J Vet Intern Med. 2007:21:700-8.

38. Kalenyak K, Isaiah A, Heilmann RM, Suchodolski JS, Burgener IA. Comparison of the intestinal mucosal microbiota in dogs diagnosed with idiopathic inflammatory bowel disease and dogs with food-responsive diarrhea before and after treatment. FEMS Microbiol Ecol. 2018;94. https://doi.org/10.1093/ femsec/fix173.

39. Minamoto Y, Dhanani N, Markel ME, Steiner JM, Suchodolski JS. Prevalence of Clostridium perfringens, Clostridium perfringens enterotoxin and dysbiosis in fecal samples of dogs with diarrhea. Vet Microbiol. 2014;174:463-73. https://doi.org/10.1016/j.vetmic.2014.10.005.

40. Ziese A-L, Suchodolski JS, Hartmann K, Busch K, Anderson A, Sarwar F, et al. Effect of probiotic treatment on the clinical course, intestinal microbiome, and toxigenic Clostridium perfringens in dogs with acute hemorrhagic diarrhea. PLoS ONE. 2018;13:e0204691. https://doi.org/10.1371/journal.pone. 0204691.

41. Moon CD, Young W, Maclean PH, Cookson AL, Bermingham EN. Metagenomic insights into the roles of Proteobacteria in the gastrointestinal microbiomes of healthy dogs and cats. Microbiologyopen. 2018;7:e00677. https://doi.org/10.1002/mbo3.677.

42. Gevers D, Kugathasan S, Denson LA, Vázquez-Baeza Y, Van Treuren W, Ren $B$, et al. The treatment-naive microbiome in new-onset Crohn's disease. Cell Host Microbe. 2014;15:382-92. https://doi.org/10.1016/j.chom.2014.02.005.

43. Islam KBMS, Fukiya S, Hagio M, Fujii N, Ishizuka S, Ooka T, et al. Bile acid is a host factor that regulates the composition of the cecal microbiota in rats. Gastroenterology. 2011:141:1773-81. https://doi.org/10.1053/j.gastro.2011.07.046.

44. Wells JE, Hylemon PB. Identification and characterization of a bile acid 7alpha-dehydroxylation operon in Clostridium sp. strain TO-931, a highly active 7alpha-dehydroxylating strain isolated from human feces. Appl Environ Microbiol. 2000;66:1107-13.

45. Kitahara M, Takamine F, Imamura T, Benno Y. Clostridium hiranonis sp. nov., a human intestinal bacterium with bile acid 7alpha-dehydroxylating activity. Int J Syst Evol Microbiol. 2001;51(Pt 1):39-44. https://doi.org/10.1099/002 07713-51-1-39.

46. Banaszkiewicz A, Kądzielska J, Gawrońska A, Pituch H, Obuch-Woszczatyński $P$, Albrecht $P$, et al. Enterotoxigenic Clostridium perfringens infection and pediatric patients with inflammatory bowel disease. J Crohns Colitis. 2014;8: 276-81. https://doi.org/10.1016/j.crohns.2013.08.018.

47. Song $Z$, Cai $Y$, Lao $X$, Wang $X$, Lin $X$, Cui $Y$, et al. Taxonomic profiling and populational patterns of bacterial bile salt hydrolase (BSH) genes based on worldwide human gut microbiome. Microbiome. 2019;7:9. https://doi.org/1 0.1186/s40168-019-0628-3.

48. Gadaleta RM, Garcia-Irigoyen O, Moschetta A. Exploration of inflammatory bowel disease in mice: chemically induced murine models of inflammatory 
bowel disease (IBD). Curr Protoc Mouse Biol. 2017;7:13-28. https://doi.org/1 $0.1002 / \mathrm{cpmo} .20$

49. Segata N, Waldron L, Ballarini A, Narasimhan V, Jousson O, Huttenhower C. Metagenomic microbial community profiling using unique clade-specific marker genes. Nat Methods. 2012;9:811-4. https://doi.org/10.1038/nmeth.2066.

50. Kitahara M, Takamine F, Imamura T, Benno Y. Assignment of Eubacterium sp. VPI 12708 and related strains with high bile acid 7alpha-dehydroxylating activity to Clostridium scindens and proposal of Clostridium hylemonae sp. nov., isolated from human faeces. Int I Syst Evol Microbiol. 2000;50(Pt 3): 971-8. https://doi.org/10.1099/00207713-50-3-971.

51. Kruis W, Kalek HD, Stellaard F, Paumgartner G. Altered fecal bile acid pattern in patients with inflammatory bowel disease. Digestion. 1986;35:189-98. https://doi.org/10.1159/000199367.

52. Giaretta PR, Rech RR, Guard BC, Blake AB, Blick AK, Steiner JM, et al. Comparison of intestinal expression of the apical sodium-dependent bile acid transporter between dogs with and without chronic inflammatory enteropathy. J Vet Intern Med. 2018:32:1918-26. https://doi.org/10.1111/jvim.15332.

53. AlShawaqfeh MK, Wajid B, Minamoto Y, Markel M, Lidbury JA, Steiner JM, et al. A dysbiosis index to assess microbial changes in fecal samples of dogs with chronic inflammatory enteropathy. FEMS Microbiol Ecol. 2017;93. https://doi.org/10.1093/femsec/fix136.

54. do Nascimento PGG, Lemos TLG, Almeida MCS, de Souza JMO, Bizerra AMC, Santiago GMP, et al. Lithocholic acid and derivatives: antibacterial activity. Steroids. 2015;104:8-15. https://doi.org/10.1016/j.steroids.2015.07.007.

55. Ward JBJ, Lajczak NK, Kelly OB, O'Dwyer AM, Giddam AK, Ní Gabhann J, et al. Ursodeoxycholic acid and lithocholic acid exert anti-inflammatory actions in the colon. Am J Physiol Gastrointest Liver Physiol. 2017;312:G550-8. https://doi.org/10.1152/ajpgi.00256.2016.

56. Vavassori P, Mencarelli A, Renga B, Distrutti E, Fiorucci S. The bile acid receptor FXR is a modulator of intestinal innate immunity. J Immunol. 2009: 183:6251-61. https://doi.org/10.4049/jimmunol.0803978.

57. Guo C, Xie S, Chi Z, Zhang J, Liu Y, Zhang L, et al. Bile acids control inflammation and metabolic disorder through inhibition of NLRP3 inflammasome. Immunity. 2016;45:802-16. https://doi.org/10.1016/j. immuni.2016.09.008.

58. Buffie CG, Bucci V, Stein RR, McKenney PT, Ling L, Gobourne A, et al. Precision microbiome reconstitution restores bile acid mediated resistance to Clostridium difficile. Nature. 2015;517:205-8. https://doi.org/10.1038/ nature13828.

59. Theriot CM, Bowman AA, Young VB. Antibiotic-induced alterations of the gut microbiota alter secondary bile acid production and allow for Clostridium difficile spore germination and outgrowth in the large intestine. mSphere. 2016;1. https://doi.org/10.1128/mSphere.00045-15.

60. Thanassi DG, Cheng LW, Nikaido H. Active efflux of bile salts by Escherichia coli. J Bacteriol. 1997;179:2512-8. https://doi.org/10.1128/ib.179.8.2512-2518.1997.

61. Ma D, Cook DN, Hearst JE, Nikaido H. Efflux pumps and drug resistance in gram-negative bacteria. Trends Microbiol. 1994;2:489-93. https://doi.org/10.1 016/0966-842X(94)90654-8.

62. Merritt ME, Donaldson JR. Effect of bile salts on the DNA and membrane integrity of enteric bacteria. J Med Microbiol. 2009;58(Pt 12):1533-41. https://doi.org/10.1099/jmm.0.014092-0.

63. Lewis BB, Carter RA, Pamer EG. Bile acid sensitivity and in vivo virulence of clinical Clostridium difficile isolates. Anaerobe. 2016;41:32-6. https://doi. org/10.1016/j.anaerobe.2016.05.010.

64. Rodrigues CM, Kren BT, Steer CJ, Setchell KD. The site-specific delivery of ursodeoxycholic acid to the rat colon by sulfate conjugation. Gastroenterology. 1995;109:1835-44.

65. Batta AK, Salen G, Holubec H, Brasitus TA, Alberts D, Earnest DL. Enrichment of the more hydrophilic bile acid ursodeoxycholic acid in the fecal water-soluble fraction after feeding to rats with colon polyps. Cancer Res. 1998;58:1684-7.

66. Dunn KA, Moore-Connors J, Maclntyre B, Stadnyk AW, Thomas NA, Noble A, et al. Early changes in microbial community structure are associated with sustained remission after nutritional treatment of pediatric Crohn's disease. Inflamm Bowel Dis. 2016;22:2853-62. https://doi.org/10.1097/MIB. 0000000000000956.

67. Bresciani F, Minamoto Y, Suchodolski JS, Galiazzo G, Vecchiato CG, Pinna C, et al. Effect of an extruded animal protein-free diet on fecal microbiota of dogs with food-responsive enteropathy. J Vet Intern Med. 2018;32:1903-10. https://doi.org/10.1111/jvim.15227.

68. Mandigers PJJ, Biourge V, van den Ingh TSGAM, Ankringa N, German AJ. A randomized, open-label, positively-controlled field trial of a hydrolyzed protein diet in dogs with chronic small bowel enteropathy. J Vet Intern Med. 2010;24:1350-7. https://doi.org/10.1111/j.1939-1676.2010.0632.x.

69. Marks SL, Laflamme DP, McAloose D. Dietary trial using a commercial hypoallergenic diet containing hydrolyzed protein for dogs with inflammatory bowel disease. Vet Ther. 2002;3:109-18.

70. Kozich JJ, Westcott SL, Baxter NT, Highlander SK, Schloss PD. Development of a dual-index sequencing strategy and curation pipeline for analyzing amplicon sequence data on the MiSeq Illumina sequencing platform. Appl Environ Microbiol. 2013;79:5112-20. https://doi.org/10.1128/AEM.01043-13.

71. Caporaso JG, Kuczynski J, Stombaugh J, Bittinger K, Bushman FD, Costello EK, et al. QIIME allows analysis of high-throughput community sequencing data. Nat Methods. 2010;7:335-6. https://doi.org/10.1038/nmeth..303.

72. Schloss PD, Westcott SL, Ryabin T, Hall JR, Hartmann M, Hollister EB, et al. Introducing mothur: open-source, platform-independent, communitysupported software for describing and comparing microbial communities Appl Environ Microbiol. 2009;75:7537-41. https://doi.org/10.1128/AEM 01541-09.

73. Edgar RC. Search and clustering orders of magnitude faster than BLAST. Bioinformatics. 2010;26:2460-1. https://doi.org/10.1093/bioinformatics/ bta461.

74. Quast C, Pruesse E, Yilmaz P, Gerken J, Schweer T, Yarza P, et al. The SILVA ribosomal RNA gene database project: improved data processing and webbased tools. Nucleic Acids Res. 2013;41(Database issue):D590-6. https://doi. org/10.1093/nar/gks1219.

75. Pruesse E, Quast C, Knittel K, Fuchs BM, Ludwig W, Peplies J, et al. SILVA: a comprehensive online resource for quality checked and aligned ribosomal RNA sequence data compatible with ARB. Nucleic Acids Res. 2007;35:718896. https://doi.org/10.1093/nar/gkm864.

76. Core Team R. R: a language and environment for statistical computing. 2017

77. Huber W, Carey VJ, Gentleman R, Anders S, Carlson M, Carvalho BS, et al. Orchestrating high-throughput genomic analysis with Bioconductor. Nat Methods. 2015;12:115-21. https://doi.org/10.1038/nmeth.3252.

78. McMurdie PJ, Holmes S. phyloseq: an R package for reproducible interactive analysis and graphics of microbiome census data. PLOS ONE. 2013;8:e61217. https://doi.org/10.1371/journal.pone.0061217.

79. Pielou EC. The measurement of diversity in different types of biological collections. J Theor Biol. 1966;13:131-44. https://doi.org/10.1016/0022-5193 (66) $90013-0$.

80. Love MI, Huber W, Anders S. Moderated estimation of fold change and dispersion for RNA-seq data with DESeq2. Genome Biol. 2014;15:550. https://doi.org/10.1186/s13059-014-0550-8.

81. Li H, Handsaker B, Wysoker A, Fennell T, Ruan J, Homer N, et al. The sequence alignment/map format and SAMtools. Bioinformatics. 2009;25: 2078-9. https://doi.org/10.1093/bioinformatics/btp352.

82. Franzosa EA, Mclver $\amalg$, Rahnavard $G$, Thompson LR, Schirmer M, Weingart $G$, et al. Species-level functional profiling of metagenomes and metatranscriptomes. Nat Methods. 2018;15:962-8. https://doi.org/10.1038/ s41592-018-0176-y.

83. Li H, Durbin R. Fast and accurate long-read alignment with BurrowsWheeler transform. Bioinformatics. 2010;26:589-95. https://doi.org/10.1093/ bioinformatics/btp698.

84. Browne HP, Forster SC, Anonye BO, Kumar N, Neville BA, Stares MD, et al. Culturing of "unculturable" human microbiota reveals novel taxa and extensive sporulation. Nature. 2016;533:543-6. https://doi.org/10.1038/ nature17645.

85. Goodman AL, Kallstrom G, Faith JJ, Reyes A, Moore A, Dantas G, et al. Extensive personal human gut microbiota culture collections characterized and manipulated in gnotobiotic mice. Proc Natl Acad Sci USA. 2011;108: 6252-7. https://doi.org/10.1073/pnas.1 102938108.

86. De Man JC, Rogosa M, Sharpe ME. A medium for the cultivation of lactobacilli. J Appl Bacteriol. 1960;23:130-135. doi:https://doi.org/10.1111/ j.1365-2672.1960.tb00188.x.

87. Ondov BD, Treangen TJ, Melsted P, Mallonee AB, Bergman NH, Koren S, et al. Mash: fast genome and metagenome distance estimation using MinHash. Genome Biol. 2016;17:132. https://doi.org/10.1186/s13059-0160997-X.

88. Lunter G, Goodson M. Stampy: a statistical algorithm for sensitive and fast mapping of Illumina sequence reads. Genome Res. 2011;21:936-9. https:/ doi.org/10.1101/gr.111120.110.

89. Friedman ES, Li Y, Shen T-CD, Jiang J, Chau L, Adorini L, et al. FXRdependent modulation of the human small intestinal microbiome by the 
bile acid derivative obeticholic acid. Gastroenterology. 2018;155:1741-1752. e5. https://doi.org/10.1053/j.gastro.2018.08.022.

90. Suzuki R, Kohno H, Sugie S, Nakagama H, Tanaka T. Strain differences in the susceptibility to azoxymethane and dextran sodium sulfate-induced colon carcinogenesis in mice. Carcinogenesis. 2006;27:162-9. https://doi.org/10.1 093/carcin/bgi205.

91. Kennedy RJ, Hoper M, Deodhar K, Erwin PJ, Kirk SJ, Gardiner KR. Interleukin 10-deficient colitis: new similarities to human inflammatory bowel disease. Br J Surg. 2000;87:1346-51. https://doi.org/10.1046/ j.1365-2168.2000.01615.x.

92. Oliveira FS, Brestelli J, Cade S, Zheng J, lodice J, Fischer S, et al. MicrobiomeDB: a systems biology platform for integrating, mining and analyzing microbiome experiments. Nucleic Acids Res. 2018;46:D684-91. https://doi.org/10.1093/nar/gkx1027.

\section{Publisher's Note}

Springer Nature remains neutral with regard to jurisdictional claims in published maps and institutional affiliations.

Ready to submit your research? Choose BMC and benefit from:

- fast, convenient online submission

- thorough peer review by experienced researchers in your field

- rapid publication on acceptance

- support for research data, including large and complex data types

- gold Open Access which fosters wider collaboration and increased citations

- maximum visibility for your research: over $100 \mathrm{M}$ website views per year

At $\mathrm{BMC}$, research is always in progress.

Learn more biomedcentral.com/submissions 